\title{
Positive psychology interventions: a meta-analysis of randomized controlled studies
}

\author{
Linda Bolier ${ }^{1 *}$, Merel Haverman², Gerben J Westerhof ${ }^{3}$, Heleen Riper $^{4,5}$, Filip Smit ${ }^{1,6}$ and Ernst Bohlmeijer ${ }^{3}$
}

\begin{abstract}
Background: The use of positive psychological interventions may be considered as a complementary strategy in mental health promotion and treatment. The present article constitutes a meta-analytical study of the effectiveness of positive psychology interventions for the general public and for individuals with specific psychosocial problems.

Methods: We conducted a systematic literature search using PubMed, Psychlnfo, the Cochrane register, and manual searches. Forty articles, describing 39 studies, totaling 6,139 participants, met the criteria for inclusion. The outcome measures used were subjective well-being, psychological well-being and depression. Positive psychology interventions included self-help interventions, group training and individual therapy.

Results: The standardized mean difference was 0.34 for subjective well-being, 0.20 for psychological well-being and 0.23 for depression indicating small effects for positive psychology interventions. At follow-up from three to six months, effect sizes are small, but still significant for subjective well-being and psychological well-being, indicating that effects are fairly sustainable. Heterogeneity was rather high, due to the wide diversity of the studies included. Several variables moderated the impact on depression: Interventions were more effective if they were of longer duration, if recruitment was conducted via referral or hospital, if interventions were delivered to people with certain psychosocial problems and on an individual basis, and if the study design was of low quality. Moreover, indications for publication bias were found, and the quality of the studies varied considerably.
\end{abstract}

Conclusions: The results of this meta-analysis show that positive psychology interventions can be effective in the enhancement of subjective well-being and psychological well-being, as well as in helping to reduce depressive symptoms. Additional high-quality peer-reviewed studies in diverse (clinical) populations are needed to strengthen the evidence-base for positive psychology interventions.

Keywords: Well-being, Depression, Positive psychology, Interventions, Effectiveness, Randomized controlled trials, Meta-analysis

\section{Background}

Over the past few decades, many psychological treatments have been developed for common mental problems and disorders such as depression and anxiety. Effectiveness has been established for cognitive behavioral therapy [1,2], problem-solving therapy [3] and interpersonal therapy [4]. Preventive and early interventions, such as the Coping with Depression course [5], the Don't Panic course [6] and Living Life to the Full

\footnotetext{
* Correspondence: Ibolier@trimbos.nl

'Department of Public Mental Health, Trimbos Institute, Netherlands Institute of Mental Health and Addiction, P.O. Box 7253500 AS, Utrecht, the Netherlands

Full list of author information is available at the end of the article
}

$[7,8]$ are also available. The existing evidence shows that the mental health care system has traditionally focused more on treatment of mental disorders than on prevention. However, it is recognized that mental health is more than just the absence of mental illness, as expressed in the World Health Organization's definition of mental health:

Mental health is a state of well-being in which the individual realizes his or her own abilities, can cope with the normal stresses of life, can work productively, and is able to make a contribution to his or her community [9].

\section{Biomed Central}


Under this definition well-being and positive functioning are core elements of mental health. It underscores that people can be free of mental illness and at the same time be unhappy and exhibit a high level of dysfunction in daily life [10]. Likewise, people with mental disorders, can be happy by coping well with their illness and enjoy a satisfactory quality of life [11]. Subjective well-being refers to a cognitive and/or affective appraisal of one's own life as a whole [12]. Psychological well-being focuses on the optimal functioning of the individual and includes concepts such as mastery, hope and purpose in life $[13,14]$. The benefits of well-being are recorded both in cross-sectional and longitudinal research and include improved productivity at work, having more meaningful relationships and less health care uptake $[15,16]$. Wellbeing is also positively associated with better physical health [17-19]. It is possible that this association is mediated by a healthy lifestyle and a healthier immune system, which buffers the adverse influence of stress [20]. In addition, the available evidence suggests that well-being reduces the risk of developing mental symptoms and disorders [21,22] and helps reduce mortality risks in people with physical disease [23].

Seligman and Csikszentmihaly's (2000) pioneered these principles of positive psychology in their well-known article entitled 'Positive psychology: An introduction', published in a special issue of the American Psychologist. They argued that a negative bias prevailed in psychology research, where the main focus was on negative emotions and treating mental health problems and disorders [24]. Although the basic concepts of well-being, happiness and human flourishing have been studied for some decades [12,25-27], there was a lack of evidence-based interventions [24]. Since the publication of Seligman and Csikszentmihaly's seminal article, the positive psychology movement has grown rapidly. The ever-expanding International Positive Psychology Association is among the most extensive research networks in the world [28] and many clinicians and coaches embrace the body of thought that positive psychology has to offer.

Consequently, the number of evaluation studies has greatly increased over the past decade. Many of these studies demonstrated the efficacy of positive psychology interventions such as counting your blessings [29,30], practicing kindness [31], setting personal goals [32,33], expressing gratitude [30,34] and using personal strengths [30] to enhance well-being, and, in some cases, to alleviate depressive symptoms [30]. Many of these interventions are delivered in a self-help format. Sin and Lyubomirsky (2009) conducted a meta-analytical review of the evidence for the effectiveness of positive psychology interventions (PPIs). Their results show that PPIs can indeed be effective in enhancing well-being $(r=0.29$, standardized mean difference Cohen's $d=0.61$ ) and help to reduce depressive symptom levels in clinical populations ( $\mathrm{r}=0.31$, Cohen's $d=0.65$ ). However, this meta-analysis had some important limitations. First, the meta-analysis included both randomized studies and quasi-experimental studies. Second, study quality was not addressed as a potential effect moderator. In recent meta-analyses, it has been shown that the treatment effects of psychotherapy have been overestimated in lower quality studies $[35,36]$. The lack of clarity in the inclusion criteria constitutes a third limitation. Intervention studies, although related to positive psychology but not strictly developed within this new framework (e.g. mindfulness, life-review) were included in the meta-analysis. However, inclusion of these studies reduces the robustness of the results for pure positive psychology interventions.

\section{Present study}

The aim of the present study is to conduct a metaanalysis of the effects of specific positive psychology interventions in the general public and in people with specific psychosocial problems. Subjective well-being, psychological well-being and depressive symptoms were the outcome measures. Potential variables moderating the effectiveness of the interventions, such as intervention type, duration and quality of the research design, were also examined. This study will add to the existing literature and the above meta-analytical review [37] by 1) only including randomized controlled studies, 2) taking the methodological quality of the primary studies into account, 3) including the most recent studies (2009 - 2012), 4) analyzing not only post-test effects but also long-term effects at follow up, and 5) applying clear inclusion criteria for the type of interventions and study design.

\section{Method}

\section{Search strategy}

A systematic literature search was carried out in PsychInfo, PubMed and the Cochrane Central Register of Controlled Trials, covering the period from 1998 (the start of the positive psychology movement) to November 2012. The search strategy was based on two key components: there should be a) a specific positive psychology intervention, and b) an outcome evaluation. The following MeSH terms and text words were used: "well-being" or "happiness" or "happy", "optimism", "positive psychology" in combination with "intervention", "treatment", "therapy" and "prevention". This was combined with terms related to outcome research: "effect", or "effic", or "outcome*", or "evaluat". We also cross-checked the references from the studies retrieved, the earlier metaanalysis of Sin \& Lyubomirsky (2009) and two other reviews of positive psychological interventions [38,39]. 
The search was restricted to peer-reviewed studies in the English language.

\section{Selection of studies}

Two reviewers (LB and $\mathrm{MH}$ ) independently selected potentially eligible studies in two phases. At the first phase, selection was based on title and abstract, and at the second phase on the full-text article. All studies identified as potentially eligible by at least one of the reviewers during the first selection phase, were re-assessed at the second selection phase. During the second phase, disagreements between the reviewers were resolved by consensus. The inter-rater reliability (kappa) was 0.90 .

The inclusion criteria were as follows:

- Examination of the effects of a positive psychology intervention. A positive psychology intervention (PPI) was defined in accordance with Sin and Lyubomirsky's (2009) article as a psychological intervention (training, exercise, therapy) primarily aimed at raising positive feelings, positive cognitions or positive behavior as opposed to interventions aiming to reduce symptoms, problems or disorders. The intervention should have been explicitly developed in line with the theoretical tradition of positive psychology (usually reported in the introduction section of an article).

- Randomization of the study subjects (randomizing individuals, not groups) and the presence of a comparator condition (no intervention, placebo, care as usual).

- Publication in a peer-reviewed journal.

- At least one of the following are measured as outcomes: well-being (subjective well-being and/or psychological well-being) or depression (diagnosis or symptoms).

- Sufficient statistics are reported to enable the calculation of standardized effect sizes.

If necessary, authors were contacted for supplementary data. We excluded studies that involved physical exercises aimed at the improvement of well-being, as well as mindfulness or meditation interventions, forgiveness therapy, life-review and reminiscence interventions. Furthermore, well-being interventions in diseased populations not explicitly grounded in positive psychology theory ('coping with disease courses') were excluded. Apart from being beyond the scope of this meta-analysis, extensive meta-analyses have already been published for these types of intervention [40-42]. This does not imply that these interventions do not have positive effects on well-being, a point which will be elaborated on in the discussion section of this paper.

\section{Data extraction}

Data extraction and study quality assessment were performed by one reviewer (LB) and independently checked by a second reviewer $(\mathrm{MH})$. Disagreements were resolved by consensus. Data were collected on design, intervention characteristics, target group, recruitment methods, delivery mode, number of sessions, attrition rates, control group, outcome measures and effect sizes (post-test and at follow up of at least 3 months). The primary outcomes in our meta-analysis were subjective well-being (SWB), psychological well-being (PWB) and depressive symptoms/depression.

The methodological quality of the included studies was assessed using a short scale of six criteria tailored to those studies and based on criteria established by the Cochrane collaboration [43]: 1) Adequacy of randomization concealment, 2) Blinding of subjects to the condition (blinding of assessors was not applicable in most cases), 3) Baseline comparability: were study groups comparable at the beginning of the study and was this explicitly assessed? (Or were adjustments made to correct for baseline imbalance using appropriate covariates), 4) Power analysis: is there an adequate power analysis and/or are there at least 50 participants in the analysis?, 5) Completeness of follow up data: clear attrition analysis and loss to follow up $<50 \%, 6)$ Handling of missing data: the use of intention-to-treat analysis (as opposed to a completers-only analysis). Each criterion was rated as 0 (study does not meet criterion) or 1 (study meets criterion). The inter-rater reliability (kappa) was 0.91 . The quality of a study was assessed as high when five or six criteria were met, medium when three or four criteria were met, and low when zero, one or two criteria were met. Along with a summary score, the aspects relating to quality were also considered individually, as results based on composite quality scales can be equivocal [44]. Table 1 shows the quality assessment for each study. The quality of the studies was scored from 1 to $5(\mathrm{M}=2.56 ; \mathrm{SD}=1.25)$. Twenty studies were rated as low, 18 were of medium quality and one study was of high quality. None of the studies met all quality criteria. The average number of participants in the analysis was rather high (17 out of 39 studies scored positive on this criterion), although none of the studies reported an adequate power analysis. Also, baseline comparability was frequently reported (26/39 studies). On the other hand, independence in the randomization procedure was seldom reported (7/39 studies) and an intention-to-treat analysis was rarely conducted (3/39 studies).

\section{Meta-analysis}

In a meta-analysis, the effects found in the primary studies are converted into a standardized effect size, which is no 
Table 1 Quality assessment per study

\begin{tabular}{|c|c|c|c|c|c|c|c|}
\hline Study & 1 & 2 & 3 & 4 & 5 & 6 & Total \\
\hline Abbott 2009 [78] & 0 & 0 & 1 & 1 & 0 & 1 & 3 \\
\hline Boehm 2011 [73] & 0 & 1 & 1 & 1 & 0 & 1 & 4 \\
\hline Buchanan 2010 [56] & 0 & 0 & 0 & 1 & 0 & 0 & 1 \\
\hline Burton 2004 [64] & 0 & 1 & 1 & 1 & 1 & 0 & 4 \\
\hline Cheavens 2006 [76] & 0 & 0 & 1 & 0 & 1 & 0 & 2 \\
\hline Emmons 2006 study 1 & 0 & 1 & 0 & 1 & 0 & 0 & 2 \\
\hline Emmons 2006 study 3 & 0 & 1 & 0 & 1 & 0 & 0 & 2 \\
\hline Fava 1998 [82] & 0 & 1 & 0 & 0 & 1 & 0 & 2 \\
\hline Fava 2005 [83] & 0 & 1 & 0 & 0 & 0 & 0 & 1 \\
\hline Feldman 2012 [60] & 0 & 0 & 0 & 1 & 0 & 0 & 1 \\
\hline Frieswijk 2005 & 0 & 0 & 0 & 1 & 0 & 0 & 1 \\
\hline Gander 2012 [74] & 0 & 1 & 1 & 1 & 0 & 0 & 3 \\
\hline Goldstein 2007 [84] & 0 & 0 & 0 & 1 & 0 & 0 & 1 \\
\hline Grant 2009 [79] & 0 & 0 & 0 & 0 & 0 & 0 & 0 \\
\hline Grant 2012 [82] & 1 & 1 & 1 & 1 & 0 & 0 & 4 \\
\hline Green 2006 [33] & 0 & 0 & 0 & 1 & 1 & 0 & 2 \\
\hline Hurley 2012 [61] & 0 & 0 & 1 & 1 & 0 & 0 & 2 \\
\hline King 2001 [66] & 0 & 1 & 0 & 0 & 1 & 0 & 2 \\
\hline Kremers 2006 [57] & 0 & 0 & 1 & 1 & 1 & 0 & 3 \\
\hline Layous 2012 [75] & 0 & 1 & 1 & 1 & 0 & 0 & 3 \\
\hline Lichter 1980 study 2 [80] & 0 & 0 & 0 & 0 & 1 & 0 & 1 \\
\hline Luthans 2008 [65] & 1 & 1 & 1 & 1 & 0 & 0 & 4 \\
\hline Luthans 2010 study 1 [72] & 0 & 1 & 1 & 1 & 1 & 0 & 4 \\
\hline Lyubomirsky 2006 study 2 [58] & 0 & 1 & 1 & 1 & 1 & 0 & 4 \\
\hline Lyubomirsky 2011 [67] & 0 & 1 & 1 & 1 & 0 & 0 & 3 \\
\hline Martinez 2010 [68] & 0 & 1 & 1 & 1 & 1 & 0 & 4 \\
\hline Mitchell 2009 [69] & 1 & 1 & 1 & 1 & 0 & 1 & 5 \\
\hline Page 2012 [62] & 1 & 0 & 1 & 0 & 0 & 0 & 2 \\
\hline Peters 2010 [70] & 0 & 1 & 1 & 1 & 1 & 0 & 4 \\
\hline Quoidbach 2009 [59] & 1 & 0 & 1 & 0 & 0 & 0 & 2 \\
\hline Schueller 2012 [63] & 1 & 0 & 1 & 1 & 0 & 0 & 3 \\
\hline Seligman 2005 [30] & 0 & 1 & 1 & 1 & 1 & 0 & 4 \\
\hline Seligman 2006 study 1 [51] & 0 & 0 & 1 & 0 & 0 & 0 & 1 \\
\hline Seligman 2006 study 2 [51] & 0 & 1 & 1 & 0 & 1 & 0 & 3 \\
\hline Shapira 2010 [55]; Mongrain 2011 [53]; Sergeant 2011 [54]; Mongrain 2012 [52] & 1 & 1 & 1 & 1 & 0 & 0 & 4 \\
\hline Sheldon 2002 [32] & 0 & 1 & 1 & 1 & 1 & 0 & 4 \\
\hline Sheldon 2006 [34] & 0 & 1 & 1 & 0 & 0 & 0 & 2 \\
\hline Spence 2007 [81] & 0 & 0 & 1 & 0 & 0 & 0 & 1 \\
\hline Wing 2006 [71] & 0 & 1 & 0 & 1 & 0 & 0 & 2 \\
\hline Total & 7 & 23 & 26 & 27 & 14 & 3 & 100 \\
\hline
\end{tabular}

Index:

$1=$ Randomization concealment.

$2=$ Blinding of subjects.

3 = Baseline comparability.

$4=$ Power analysis or $\mathrm{N}>=50$.

$5=$ Completeness of follow up data.

$6=$ Intention-to-treat analysis. 
longer placed on the original measurement scale, and can therefore be compared with measures from other scales. For each study, we calculated effect sizes (Cohen's $d$ ) by subtracting the average score of the experimental group (Me) from the average score of the control group (Mc), and dividing the result by the pooled standard deviations of both groups. This was done at post-test because randomization usually results in comparable groups across conditions at baseline. However, if baseline differences on outcome variables did exist despite the randomization, d's were calculated on the basis of pre- post-test differences: by calculating the standardized pre- post change score for the experimental group (de) and the control group (dc) and subsequently calculating their difference as $\Delta \mathrm{d}=\mathrm{de}-\mathrm{dc}$. For example, an effect size of 0.5 indicates that the mean of the experimental group is half a standard unit (standard deviation) larger than the mean of the control group. From a clinical perspective, effect sizes of $0.56-1.2$ can be interpreted as large, while effect sizes of $0.33-0.55$ are of medium size, and effects of $0-0.32$ are small [45].

In the calculation of effect sizes for depression, we used instruments that explicitly measure depression (e.g. the Beck Depression Inventory, or the Center for Epidemiological Studies Depression Scale). For subjective and psychological well-being, we also used instruments related to the construct of well-being (such as positive affect for SWB and hope for PWB). If more than one measure was used for SWB, PWB or depression, the mean of the effect sizes was calculated, so that each study outcome had one effect size. If more than one experimental group was compared with a control condition in a particular study, the number of subjects in the control groups was evenly divided across the experimental groups so that each subject was used only once in the meta-analysis.

To calculate pooled mean effect sizes, we used Comprehensive Meta-Analysis (CMA, Version 2.2.064). Due to the diversity of studies and populations, a common effect size was not assumed and we expected considerable heterogeneity. Therefore, it was decided a priori to use the 'random effects model'. Effect sizes may differ under this model, not only because of random error within studies (as in the fixed effects model), but also as a result of true variation in effect sizes between studies. The outcomes of the random effects model are conservative in that their 95\% Confidence Intervals (CIs) are often broad, thus reducing the likelihood of type-II errors.

We tested for the presence of heterogeneity with two indicators. First, we calculated the Q-statistic. A significant $\mathrm{Q}$ rejects the null-hypothesis of homogeneity and indicates that the true effect size probably does vary from study to study. Second, the $\mathrm{I}^{2}$-statistic was calculated. This is a percentage indicating the study-to-study dispersion due to real differences, over and above random sampling error. A value of $0 \%$ indicates an absence of dispersion, and larger values show increasing levels of heterogeneity where $25 \%$ can be considered as low, $50 \%$ as moderate and $75 \%$ as a high level of heterogeneity [46].

Owing to the expected high level of heterogeneity, all studies were taken into account. Outliers were considered, but not automatically removed from the meta-analysis. The procedure of removing outliers which are outside the confidence interval of the pooled effect size is advised when a common effect size is assumed. However, in our meta-analysis, high dispersion was expected and therefore only the exclusion of Cohen's $d>2.5$ from the final sample was planned.

Subgroup analyses were performed by testing differences in Cohen's d's between subgroups. Six potential moderators were determined based on previous research and the characteristics of the investigated interventions and studies: 1) Self-selected sample/not selfselected: did the participants know that the aim of the intervention was to make them feel better?; 2) Duration: less than four weeks, four to eight weeks, or more than eight weeks; 3) Type of intervention: selfhelp, group intervention, or individual therapy; 4) Recruitment method: community (in a community center, local newspapers), internet, by referral/hospital, at university; 5) Psychosocial problems (Yes/none): was the data based on a group with certain psychosocial problems or was the study open to everyone?; 6) Quality rating: low (score 1 or 2), medium (score 3 or 4 ) or high (score 5 or 6 ). The impact of the duration and quality ratings was also assessed using meta-regression.

Results of meta-analysis may be biased due to the fact that studies with non-significant or negative results are less likely to be published in peer-reviewed journals [47]. In order to address this issue, we used three indices: funnel plots, the Orwin's fail-safe number and the Trim and Fill method. A funnel plot is a graph of effect size against study size. When publication bias is absent, the observed studies are expected to be distributed symmetrically around the pooled effect size. The Orwin's fail-safe number indicates the number of nonsignificant unpublished studies needed to reduce the overall significant effect to non-significance (according to a self-stated criterium) [48]. The effect size can be considered to be robust if the number of studies required to reduce the overall effect size to a nonsignificant level exceeds $5 \mathrm{~K}+10$, where $\mathrm{K}$ is the number of studies included. If asymmetry is found in the funnel plot, the Trim and Fill method adjusts the pooled effect size for the outcomes of missing studies [49]. Imputing missing studies restores the symmetry in the funnel plot and an adjusted effect size can be calculated. 
For the reporting of the results of this meta-analysis, we applied Preferred Reporting Items for Systematic Reviews and Meta-Analyses (PRISMA) guidelines [50].

\section{Results}

\section{Description of studies}

The selection process is illustrated in Figure 1. First, 5,335 titles were retrieved from databases and 55 titles were identified through searching the reference list accompanying the meta-analysis by Sin and Lyubomirsky (2009) [37] as well as two other literature reviews of positive psychological interventions $[38,39]$. After reviewing the titles and abstracts and removing duplicates, 84 articles were identified as being potentially eligible for inclusion in our study. Of these 84 articles, 40 articles in which 39 studies were described, met our inclusion criteria (of these, 17 articles describing 19 studies were also included in the metaanalysis by Sin and Lyubomirsky, 2009). In two articles
[29,51] two studies were described, and one study [52-55] was published in four articles.

The characteristics of the studies included are described in Table 2. The studies evaluated 6,139 subjects, 4,043 in PPI groups and 2,096 in control groups. Ten studies compared a PPI with a no-intervention control group [29,51,56-63], 17 studies compared a PPI with a placebo intervention [29,30,32,34,52-55,64-75], seven studies with a waiting list control group [33,76-81] and five studies with another active intervention (care as usual) [51,82-85]. A minority of seven studies [51,57,76,77,82,83] applied inclusion criteria to target a specific group with psychosocial problems such as depression and anxiety symptoms. Half of the studies, 19 in total, recruited the subjects (not necessarily students) through university [29,32,34,51,56,58$61,64-68,70,72,75,80,85]$. In seven studies subjects were recruited in the community $[33,57,71,73,76,77,81]$, in four studies by referral from a practitioner or hospital

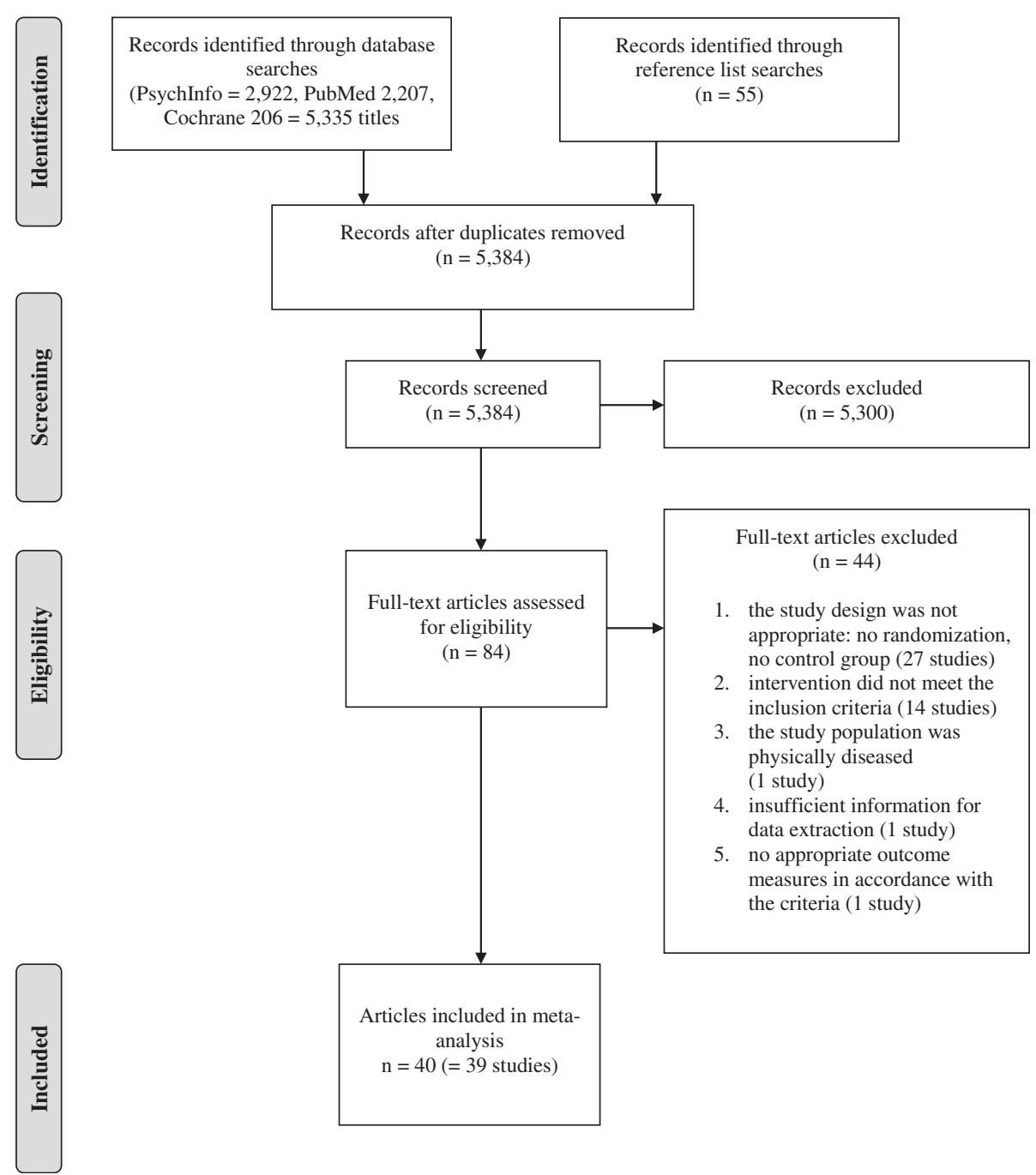

Figure 1 Flow diagram. 
Table 2 Characteristics of randomized controlled trials examining the effects of positive psychology interventions

\begin{tabular}{|c|c|c|c|c|c|c|c|c|c|c|c|c|}
\hline Author & Intervention & $\begin{array}{l}\text { Session } \\
\text { (number), } \\
\text { duration }\end{array}$ & $\begin{array}{l}\text { Mean age } \\
\text { (range or } \\
\text { SD) }\end{array}$ & Delivery & Recruitment & $\begin{array}{l}\text { Self- } \\
\text { selection }\end{array}$ & $\begin{array}{l}\text { Psychosocial problems / } \\
\text { inclusion criteria }\end{array}$ & $\begin{array}{l}\text { Control } \\
\text { Group }\end{array}$ & $\begin{array}{l}\mathrm{N} \\
\text { analyzed } \\
\text { (post } \\
\text { test) }\end{array}$ & $\begin{array}{l}\text { Attrition } \\
\text { rate, \% } \\
\text { (post test) }\end{array}$ & $\begin{array}{l}\text { Outcome } \\
\text { measures }\end{array}$ & $\begin{array}{l}\text { Follow-up } \\
\text { (min. } \\
3 \text { months) }\end{array}$ \\
\hline $\begin{array}{l}\text { Abbott } 2009 \\
{[78]}\end{array}$ & ResilienceOnline & $7,10 \mathrm{w}$ & 43 & Self-help & Organization & $\begin{array}{l}\text { Self- } \\
\text { selected }\end{array}$ & None & Waiting list & $\begin{array}{l}\mathrm{Ne}=26 \\
\mathrm{Nc}=27\end{array}$ & $41.5 \%$ & $\begin{array}{l}\text { PWB: AHI } \\
\text { DEP: } \\
\text { DASS-21 }\end{array}$ & - \\
\hline $\begin{array}{l}\text { Boehm } 2011 \\
\text { [73] }\end{array}$ & $\begin{array}{l}\text { Optimism and } \\
\text { gratitude exercise }\end{array}$ & $6,6 w$ & $35.6(11.4)$ & Self-help & Community & $\begin{array}{l}\text { Self- } \\
\text { selected }\end{array}$ & None & Placebo & $\begin{array}{l}\mathrm{Ne}=146 \\
\mathrm{Nc}=74\end{array}$ & $?$ & SWB: SWLS & - \\
\hline $\begin{array}{l}\text { Buchanan } \\
2010[56]\end{array}$ & Doing acts of kindness & $10,10 d$ & $26(18-60)$ & Self-help & University & $\begin{array}{l}\text { Self- } \\
\text { selected }\end{array}$ & None & $\begin{array}{l}\text { No } \\
\text { intervention }\end{array}$ & $\begin{array}{l}\mathrm{Ne}=28 \\
\mathrm{Nc}=28\end{array}$ & $0 \%(? \mathrm{nr})$ & SWB: SWLS & - \\
\hline $\begin{array}{l}\text { Burton } 2004 \\
\text { [64] }\end{array}$ & $\begin{array}{l}\text { Writing about positive } \\
\text { experiences }\end{array}$ & $3,3 d$ & $\begin{array}{l}\text { College- } \\
\text { based } \\
\text { sample } \\
18.6(0.95)\end{array}$ & Self-help & University & $\begin{array}{l}\text { Not self- } \\
\text { selected }\end{array}$ & None & Placebo & $\begin{array}{l}\mathrm{Ne}=48 \\
\mathrm{Nc}=42\end{array}$ & $0 \%$ & SWB: PA & - \\
\hline $\begin{array}{l}\text { Cheavens } \\
2006[76]\end{array}$ & Hope therapy & $8,8 w$ & $49(32-64)$ & Group & Community & $\begin{array}{l}\text { Self- } \\
\text { selected }\end{array}$ & Inclusion criteria unclear & Waiting list & $\begin{array}{l}\mathrm{Ne}=17 \\
\mathrm{Nc}=15\end{array}$ & $\begin{array}{l}12 \% \\
22 \% \\
\text { T: } 18 \%\end{array}$ & $\begin{array}{l}\text { PWB: SHS } \\
\text { DEP: CES- } \\
\text { D }\end{array}$ & - \\
\hline $\begin{array}{l}\text { Emmons } \\
\text { 2006 study } 1 \\
\text { [29] }\end{array}$ & $\begin{array}{l}\text { Practising gratitude by } \\
\text { counting one's } \\
\text { blessings }\end{array}$ & $10,10 w$ & U (students) & Self-help & University & $\begin{array}{l}\text { Not self- } \\
\text { selected }\end{array}$ & None & Placebo & $\begin{array}{l}\mathrm{Ne}=65 \\
\mathrm{Nc}=67\end{array}$ & T: $4 \%$ & $\begin{array}{l}\text { SWB: Life } \\
\text { as a } \\
\text { whole, } \\
\text { upcoming } \\
\text { week, PA }\end{array}$ & - \\
\hline $\begin{array}{l}\text { Emmons } \\
2006 \text { study } 3 \\
\text { [29] }\end{array}$ & $\begin{array}{l}\text { Practising gratitude by } \\
\text { counting one's } \\
\text { blessings }\end{array}$ & $21,3 w$ & $49(22-77)$ & Self-help & $\begin{array}{l}\text { Referral/ } \\
\text { hospital }\end{array}$ & $\begin{array}{l}\text { Not self- } \\
\text { selected }\end{array}$ & None & $\begin{array}{l}\text { No } \\
\text { intervention }\end{array}$ & $\begin{array}{l}\mathrm{Ne}=33 \\
\mathrm{Nc}=32\end{array}$ & $0 \%$ (? nr) & $\begin{array}{l}\text { SWB: Life } \\
\text { as a } \\
\text { whole, } \\
\text { upcoming } \\
\text { week, PA } \\
\text { (self-report } \\
\text { and } \\
\text { observed) }\end{array}$ & - \\
\hline $\begin{array}{l}\text { Fava } 1998 \\
\text { [82] }\end{array}$ & Well-being therapy & $8,16 w$ & $28.4(6.5)$ & Individual & $\begin{array}{l}\text { Referral// } \\
\text { hospital }\end{array}$ & $\begin{array}{l}\text { Self- } \\
\text { selected }\end{array}$ & $\begin{array}{l}\text { Diagnosis of MDD or AD, } \\
\text { succesful response to } \\
\text { treatment }\end{array}$ & TAU & $\begin{array}{l}\mathrm{Ne}=10 \\
\mathrm{Nc}=10\end{array}$ & $0 \%$ & $\begin{array}{l}\text { PWB: } \\
\text { RPWB DEP: } \\
\text { CID, SQ } \\
\text { subscale }\end{array}$ & - \\
\hline $\begin{array}{l}\text { Fava } 2005 \\
{[83]}\end{array}$ & Well-being therapy & $8,16 w$ & $41.9(12)$ & Individual & $\begin{array}{l}\text { Referral/ } \\
\text { hospital }\end{array}$ & $\begin{array}{l}\text { Self- } \\
\text { selected }\end{array}$ & Diagnosis of GAD & TAU & $\begin{array}{l}\mathrm{Ne}=8 \\
\mathrm{Nc}=8\end{array}$ & $20 \%$ & $\begin{array}{l}\text { PWB: } \\
\text { RPWB DEP: } \\
\text { CID, SQ } \\
\text { subscale }\end{array}$ & $\begin{array}{l}1 \text { yr (not in } \\
\text { study) }\end{array}$ \\
\hline $\begin{array}{l}\text { Feldman } \\
2012[60]\end{array}$ & $\begin{array}{l}\text { Hopeful goal-directed } \\
\text { thinking }\end{array}$ & $1,1 d$ & $\begin{array}{l}18.7(18- \\
22)\end{array}$ & Group & University & $\begin{array}{l}\text { Not self- } \\
\text { selected }\end{array}$ & None & $\begin{array}{l}\text { No } \\
\text { intervention }\end{array}$ & $\begin{array}{l}\mathrm{Ne}=37 \\
\mathrm{Nc}=29\end{array}$ & $24.7 \%$ & $\begin{array}{l}\text { PWB: } \\
\text { GSHS, PIL }\end{array}$ & \\
\hline \multirow[t]{2}{*}{$\begin{array}{l}\text { Frieswijk } \\
2005[77]\end{array}$} & $\begin{array}{l}\text { Self-management } \\
\text { positive bibliotherapy }\end{array}$ & $5,10 \mathrm{w}$ & $72.9(6.2)$ & Self-help & Community & $\begin{array}{l}\text { Self- } \\
\text { selected }\end{array}$ & $\begin{array}{l}\text { Slightly or moderately frail } \\
(>=65 \mathrm{GFI})\end{array}$ & Waiting list & $\begin{array}{l}\mathrm{Ne}=79 \\
\mathrm{Nc}=86\end{array}$ & $\begin{array}{l}18.4 \% \\
10.4 \% \\
\text { T: } 14.5 \%\end{array}$ & $\begin{array}{l}\text { SWB: SPF- } \\
\text { IL PWB: } \\
\text { MS }\end{array}$ & $6 \mathrm{~m}$ \\
\hline & & & & Self-help & & & None & Placebo & & $74 \%$ & & $6 \mathrm{~m}$ \\
\hline
\end{tabular}


Table 2 Characteristics of randomized controlled trials examining the effects of positive psychology interventions (Continued)

\begin{tabular}{|c|c|c|c|c|c|c|c|c|c|c|c|c|}
\hline $\begin{array}{l}\text { Gander } 2012 \\
\text { [74] }\end{array}$ & $\begin{array}{l}9 \text { exercises: gratitude } \\
\text { visit three good things } \\
\text { ( } 1 \text { and } 2 \text { weeks), } \\
\text { strengths, three funny } \\
\text { things, social exercises }\end{array}$ & $\begin{array}{l}7,1 \mathrm{w} 14, \\
2 \mathrm{w}\end{array}$ & $\begin{array}{l}44.9 \\
(10.07)\end{array}$ & & $\begin{array}{l}\text { Internet, } \\
\text { magazine }\end{array}$ & $\begin{array}{l}\text { Self- } \\
\text { selected }\end{array}$ & & & $\begin{array}{l}\mathrm{Ne}=559 \\
\mathrm{Nc}=63\end{array}$ & & $\begin{array}{l}\text { PWB: AHI } \\
\text { DEP: CES- } \\
\text { D }\end{array}$ & \\
\hline $\begin{array}{l}\text { Goldstein } \\
2007 \text { [84] }\end{array}$ & $\begin{array}{l}\text { Cultivating sacred } \\
\text { moments }\end{array}$ & $15,3 w$ & $(22-44)$ & Self-help & Internet & $\begin{array}{l}\text { Self- } \\
\text { selected }\end{array}$ & None & TAU & $\begin{array}{l}\mathrm{Ne}=35 \\
\mathrm{Nc}=38\end{array}$ & $\begin{array}{l}14.6 \% \\
9.5 \% \\
\text { T: } 12.0 \%\end{array}$ & $\begin{array}{l}\text { SWB: SWLS } \\
\text { PWB: } \\
\text { RPWB }\end{array}$ & - \\
\hline $\begin{array}{l}\text { Grant } 2009 \\
\text { [79] }\end{array}$ & Executive coaching & $6,8-10 w$ & 49.8 & $\begin{array}{l}\text { Group } \\
\text { and } \\
\text { individual }\end{array}$ & Organization & $\begin{array}{l}\text { Self- } \\
\text { selected }\end{array}$ & None & Waiting list & $\begin{array}{l}\mathrm{Ne}=21 \\
\mathrm{Nc}=20\end{array}$ & $18 \%$ & $\begin{array}{l}\text { DEP: } \\
\text { DASS-21 }\end{array}$ & - \\
\hline $\begin{array}{l}\text { Grant } 2012 \\
{[85]}\end{array}$ & $\begin{array}{l}\text { Solution-focused } \\
\text { coaching }\end{array}$ & $1,1 d$ & $20.5(5.4)$ & Self-help & University & $\begin{array}{l}\text { Self- } \\
\text { selected }\end{array}$ & None & TAU & $\begin{array}{l}\mathrm{Ne}=117 \\
\mathrm{Nc}=108\end{array}$ & $0 \%(? \mathrm{nr})$ & SWB: PA & - \\
\hline $\begin{array}{l}\text { Green } 2006 \\
\text { [33] }\end{array}$ & $\begin{array}{l}\text { Life coaching and } \\
\text { attainment of goals }\end{array}$ & $10,10 w$ & $\begin{array}{l}42.7(18- \\
60)\end{array}$ & Group & Community & $\begin{array}{l}\text { Self- } \\
\text { selected }\end{array}$ & None & Waiting list & $\begin{array}{l}\mathrm{Ne}=25 \\
\mathrm{Nc}=25\end{array}$ & $\begin{array}{l}10.7 \% \\
10.7 \% \\
\text { T: } 10.7 \%\end{array}$ & $\begin{array}{l}\text { SWB: } \\
\text { SWLS, PA } \\
\text { PWB: } \\
\text { RPWB, SHS }\end{array}$ & - \\
\hline $\begin{array}{l}\text { Hurley } 2012 \\
\text { [61] }\end{array}$ & Savoring the moment & $14,2 w$ & $19.5(2.06)$ & $\begin{array}{l}\text { Group / } \\
\text { Self-help }\end{array}$ & University & $\begin{array}{l}\text { Not self- } \\
\text { selected }\end{array}$ & None & $\begin{array}{l}\text { No } \\
\text { intervention }\end{array}$ & $\begin{array}{l}\mathrm{Ne}=94 \\
\mathrm{Nc}=99\end{array}$ & $\begin{array}{l}37.7 \% \\
39.6 \% \\
\text { T: } 38.7 \%\end{array}$ & $\begin{array}{l}\text { SWB: PA } \\
\text { DEP: BDI }\end{array}$ & - \\
\hline $\begin{array}{l}\text { King } 2001 \\
{[66]}\end{array}$ & $\begin{array}{l}\text { Writing about best } \\
\text { possible selves }\end{array}$ & $4,4 d$ & $21(18-42)$ & Self-help & University & $\begin{array}{l}\text { Not self- } \\
\text { selected }\end{array}$ & None & Placebo & $\begin{array}{l}\mathrm{Ne}=19 \\
\mathrm{Nc}=16\end{array}$ & $0 \%$ & SWB: PA & - \\
\hline $\begin{array}{l}\text { Kremers } \\
2006[57]\end{array}$ & $\begin{array}{l}\text { Self-management } \\
\text { positive group course }\end{array}$ & $6,6 w$ & $64.3(7)$ & Group & Community & $\begin{array}{l}\text { Self- } \\
\text { selected }\end{array}$ & Single and lonely & $\begin{array}{l}\text { No } \\
\text { intervention }\end{array}$ & $\begin{array}{l}\mathrm{Ne}=46 \\
\mathrm{Nc}=73\end{array}$ & $\begin{array}{l}17.0 \% \\
7.6 \% \\
\text { T: } 16.2 \%\end{array}$ & $\begin{array}{l}\text { SWB: SPF- } \\
\text { IL }\end{array}$ & $6 \mathrm{~m}$ \\
\hline $\begin{array}{l}\text { Layous } 2012 \\
\text { [75] }\end{array}$ & $\begin{array}{l}\text { Best possible selves } \\
\text { exercise }\end{array}$ & $4,4 w$ & $19.1(1.8)$ & $\begin{array}{l}\text { Self-help } \\
\text { Group }\end{array}$ & University & $\begin{array}{l}\text { Not self- } \\
\text { selected }\end{array}$ & None & Placebo & $\begin{array}{l}\mathrm{Ne}=80 \\
\mathrm{Nc}=37\end{array}$ & $?$ & $\begin{array}{l}\text { SWB: PA } \\
\text { PWB: NS }\end{array}$ & - \\
\hline $\begin{array}{l}\text { Lichter } 1980 \\
\text { study } 2 \text { [80] }\end{array}$ & $\begin{array}{l}\text { Rehearsal of positive } \\
\text { statements }\end{array}$ & $14,2 w$ & $\begin{array}{l}\text { U } \\
\text { (students) }\end{array}$ & Individual & University & $\begin{array}{l}\text { Not self- } \\
\text { selected }\end{array}$ & None & Waiting list & $\begin{array}{l}\mathrm{Ne}=25 \\
\mathrm{Nc}=23\end{array}$ & $0 \%(? \mathrm{nr})$ & $\begin{array}{l}\text { SWB: AF1 } \\
\text { DEP: BDI }\end{array}$ & - \\
\hline $\begin{array}{l}\text { Luthans } \\
2008[65]\end{array}$ & $\begin{array}{l}\text { Online well-being } \\
\text { program (PsyCap) }\end{array}$ & $2,2 w$ & 32.2 & Self-help & University & $\begin{array}{l}\text { Self- } \\
\text { selected }\end{array}$ & None & Placebo & $\begin{array}{l}\mathrm{Ne}=187 \\
\mathrm{Nc}=177\end{array}$ & $\begin{array}{l}6.0 \% \\
4.8 \% \\
\text { T: } 5.5 \%\end{array}$ & PWB: PCQ & - \\
\hline $\begin{array}{l}\text { Luthans } \\
2010 \text { study } 1 \\
\text { [72] }\end{array}$ & PsyCap training & $1,2 \mathrm{~h}$ & $21.1(2.66)$ & Group & University & $\begin{array}{l}\text { Not self- } \\
\text { selected }\end{array}$ & None & Placebo & $\begin{array}{l}\mathrm{Ne}=153 \\
\mathrm{Nc}=89)\end{array}$ & $0 \%$ & PCQ & - \\
\hline $\begin{array}{l}\text { Lyubomirsky } \\
2006 \text { study } 2 \\
\text { [58] }\end{array}$ & $\begin{array}{l}\text { Thinking about } \\
\text { positive life } \\
\text { experiences }\end{array}$ & $3,3 d$ & $19.5(2.6)$ & Self-help & University & $\begin{array}{l}\text { Not self- } \\
\text { selected }\end{array}$ & None & $\begin{array}{l}\text { No } \\
\text { intervention }\end{array}$ & $\begin{array}{l}\mathrm{Ne}=26 \\
\mathrm{Nc}=36\end{array}$ & $0 \%$ & $\begin{array}{l}\text { SWB: } \\
\text { SWLS, PA }\end{array}$ & - \\
\hline $\begin{array}{l}\text { Lyubomirsky } \\
2011 \text { [67] }\end{array}$ & $\begin{array}{l}\text { Expressing optimism } \\
\text { or gratitude }\end{array}$ & $8,8 w$ & $\begin{array}{l}19.7(18- \\
46)\end{array}$ & Self-help & University & $\begin{array}{l}\text { Self- } \\
\text { selected }\end{array}$ & None & Placebo & $\begin{array}{l}\mathrm{Ne}=218 \\
\mathrm{Nc}=101\end{array}$ & T: $10.1 \%$ & $\begin{array}{l}\text { SWB: PLA, } \\
\text { SWLS, SHS } \\
\text { (2) }\end{array}$ & $6 \mathrm{~m}$ \\
\hline
\end{tabular}


Table 2 Characteristics of randomized controlled trials examining the effects of positive psychology interventions (Continued)

\begin{tabular}{|c|c|c|c|c|c|c|c|c|c|c|c|c|}
\hline $\begin{array}{l}\text { Martinez } \\
2010[68]\end{array}$ & $\begin{array}{l}\text { Practising gratitude by } \\
\text { counting one's } \\
\text { blessings }\end{array}$ & $14,2 w$ & $20.7(1.5)$ & Self-help & University & $\begin{array}{l}\text { Not self- } \\
\text { selected }\end{array}$ & None & Placebo & $\begin{array}{l}\mathrm{Ne}=41 \\
\mathrm{Nc}=34\end{array}$ & $34.0 \%$ & $\begin{array}{l}\text { SWB: PA, } \\
\text { GA (self- } \\
\text { report and } \\
\text { observed) }\end{array}$ & - \\
\hline $\begin{array}{l}\text { Mitchell } \\
2009 \text { [69] }\end{array}$ & $\begin{array}{l}\text { Online intervention } \\
\text { Use your strenghts in } \\
\text { a new way }\end{array}$ & $3,3 w$ & $37(18-62)$ & Self-help & Internet & $\begin{array}{l}\text { Self- } \\
\text { selected }\end{array}$ & None & Placebo & $\begin{array}{l}\mathrm{Ne}=48 \\
\mathrm{Nc}=54\end{array}$ & $\begin{array}{l}64.6 \% \\
57.4 \% \\
\text { T: } 60.8 \%\end{array}$ & $\begin{array}{l}\text { SWB: PWI- } \\
\text { A, SWLS, } \\
\text { PA PWB: } \\
\text { OTH DEP: } \\
\text { DASS-21 }\end{array}$ & $3 \mathrm{~m}$ \\
\hline $\begin{array}{l}\text { Page } 2012 \\
\text { [62] }\end{array}$ & $\begin{array}{l}\text { Working for Wellness } \\
\text { Program }\end{array}$ & $6,6 w$ & $39.7(10.0)$ & Group & Organization & $\begin{array}{l}\text { Self- } \\
\text { selected }\end{array}$ & None & $\begin{array}{l}\text { No } \\
\text { intervention }\end{array}$ & $\begin{array}{l}\mathrm{Ne}=13 \\
\mathrm{Nc}=10\end{array}$ & $\begin{array}{l}58.1 \% \\
66.7 \% \\
\text { T: } 62.3 \%\end{array}$ & $\begin{array}{l}\text { SWB: } \\
\text { SWLS, PA } \\
\text { PWB: } \\
\text { SPWB }\end{array}$ & 6 month \\
\hline $\begin{array}{l}\text { Peters } 2010 \\
\text { [70] }\end{array}$ & $\begin{array}{l}\text { Positive future } \\
\text { thinking }\end{array}$ & $1,1 d$ & $\begin{array}{l}29.7(21- \\
50)\end{array}$ & Self-help & University & $\begin{array}{l}\text { Not self- } \\
\text { selected }\end{array}$ & None & Placebo & $\begin{array}{l}\mathrm{Ne}=44 \\
\mathrm{Nc}=38\end{array}$ & $0 \%$ & SWB: PA & - \\
\hline $\begin{array}{l}\text { Quoidbach } \\
2009 \text { [59] }\end{array}$ & $\begin{array}{l}\text { Projecting a positive } \\
\text { self in the future }\end{array}$ & $14,2 w$ & 32.5 & Self-help & University & $\begin{array}{l}\text { Not self- } \\
\text { selected }\end{array}$ & None & $\begin{array}{l}\text { No } \\
\text { intervention }\end{array}$ & $\begin{array}{l}\mathrm{Ne}=15 \\
\mathrm{Nc}=57\end{array}$ & $\mathrm{~T}: 49.5 \%$ & $\begin{array}{l}\text { SWB: SHS } \\
\text { (2) }\end{array}$ & - \\
\hline $\begin{array}{l}\text { Schueller } \\
2012[63]\end{array}$ & $\begin{array}{l}\text { Package of 2, } 4 \text { or } 6 \\
\text { positive psychology } \\
\text { exercises (active- } \\
\text { constructive } \\
\text { responding, gratitude } \\
\text { visit, life summary, } \\
\text { three good things, } \\
\text { savoring, strengths) }\end{array}$ & $\begin{array}{l}14,2 w 28 \\
4 w 42,6 w\end{array}$ & $42.4(12.1)$ & Self-help & Internet & $\begin{array}{l}\text { Self- } \\
\text { selected }\end{array}$ & None & $\begin{array}{l}\text { No } \\
\text { intervention }\end{array}$ & $\begin{array}{l}\mathrm{Ne}=457 \\
\mathrm{Nc}=204\end{array}$ & $\begin{array}{l}54.7 \% \\
42.5 \% \\
\text { T: } 55.4 \%\end{array}$ & $\begin{array}{l}\text { DEP: CES- } \\
\text { D }\end{array}$ & - \\
\hline $\begin{array}{l}\text { Seligman } \\
2005 \text { [30] }\end{array}$ & $\begin{array}{l}\text { Strenghts excercises } \\
\text { (2), gratitude (1), } \\
\text { positive thinking (2) }\end{array}$ & $7,1 w$ & $\begin{array}{l}64 \% \\
\text { between5- } \\
54\end{array}$ & Self-help & Internet & $\begin{array}{l}\text { Self- } \\
\text { selected }\end{array}$ & None & Placebo & $\begin{array}{l}\mathrm{Ne}=341 \\
\mathrm{Nc}=70\end{array}$ & T: $28.8 \%$ & $\begin{array}{l}\text { PWB: SHI } \\
\text { DEP: CES- } \\
\text { D }\end{array}$ & $6 \mathrm{~m}$ \\
\hline $\begin{array}{l}\text { Seligman } \\
2006 \text { study } 1 \\
\text { [51] }\end{array}$ & $\begin{array}{l}\text { Group positive } \\
\text { psychotherapy }\end{array}$ & $6,6 w$ & $\begin{array}{l}\text { U } \\
\text { (students) }\end{array}$ & Group & University & $\begin{array}{l}\text { Self- } \\
\text { selected }\end{array}$ & $\begin{array}{l}\text { Mild to moderate } \\
\text { depressive symptoms } \\
\text { (BDI 10-24) }\end{array}$ & $\begin{array}{l}\text { No } \\
\text { intervention }\end{array}$ & $\begin{array}{l}\mathrm{Ne}=14 \\
\mathrm{Nc}=20\end{array}$ & $\begin{array}{l}26.3 \% \\
4.8 \% \\
\text { T: } 15.0 \%\end{array}$ & $\begin{array}{l}\text { SWB: SWLS } \\
\text { DEP: BDI }\end{array}$ & $\begin{array}{l}3,6 \\
\text { (in study), } 12 \mathrm{~m}\end{array}$ \\
\hline $\begin{array}{l}\text { Seligman } \\
2006 \text { study } 2 \\
{[51]}\end{array}$ & $\begin{array}{l}\text { Individual positive } \\
\text { psychotherapy }\end{array}$ & $14,12 W$ & U (adults) & Individual & $\begin{array}{l}\text { Referral/ } \\
\text { hospital }\end{array}$ & $\begin{array}{l}\text { Self- } \\
\text { selected }\end{array}$ & Clinical diagnosis of MDD & TAU & $\begin{array}{l}\mathrm{Ne}=11 \\
\mathrm{Nc}=9\end{array}$ & $\begin{array}{l}15.4 \% \\
40.0 \% \\
\text { T: } 28.6 \%\end{array}$ & $\begin{array}{l}\text { SWB: SWLS } \\
\text { PWB: PPTI } \\
\text { DEP: } \\
\text { HRSD, } \\
\text { ZSRS }\end{array}$ & - \\
\hline $\begin{array}{l}\text { Shapira } 2010 \\
\text { [55]; } \\
\text { Mongrain } \\
2011 \text { [53]; } \\
\text { Sergeant } \\
2011 \text { [54]; } \\
\text { Mongrain } \\
2012 \text { [52] }\end{array}$ & $\begin{array}{l}\text { Three good things, } \\
\text { signature strengths, } \\
\text { self-compassion, } \\
\text { optimism, } \\
\text { compassionate action, } \\
\text { gratitude intervention }\end{array}$ & $7,1 w$ & $34(11.8)$ & Self-help & Internet & $\begin{array}{l}\text { Self- } \\
\text { selected }\end{array}$ & None & Placebo & $\begin{array}{l}\mathrm{Ne}=804 \\
\mathrm{Nc}=138\end{array}$ & $75 \%$ & $\begin{array}{l}\text { PWB: SHI } \\
\text { DEP: CES- } \\
\text { D }\end{array}$ & $\begin{array}{l}3 \\
\text { (in study), } 6 \mathrm{~m}\end{array}$ \\
\hline
\end{tabular}


Table 2 Characteristics of randomized controlled trials examining the effects of positive psychology interventions (Continued)

\begin{tabular}{|c|c|c|c|c|c|c|c|c|c|c|c|c|}
\hline $\begin{array}{l}\text { Sheldon } \\
2002[32]\end{array}$ & Goal-training program & $2,2 w$ & $\begin{array}{l}\text { U } \\
\text { (students) }\end{array}$ & Group & University & $\begin{array}{l}\text { Not self- } \\
\text { selected }\end{array}$ & None & Placebo & $\begin{array}{l}\mathrm{Ne}=36 \\
\mathrm{Nc}=42\end{array}$ & T: $13.3 \%$ & $\begin{array}{l}\text { SWB: PA } \\
\text { PWB: } \\
\text { RPWB }\end{array}$ & - \\
\hline $\begin{array}{l}\text { Sheldon } \\
2006 \text { [34] }\end{array}$ & $\begin{array}{l}\text { Gratitude or } \\
\text { visualizing positive self }\end{array}$ & $14,2 w$ & $\begin{array}{l}U \\
\text { (students) }\end{array}$ & Self-help & University & $\begin{array}{l}\text { Not self- } \\
\text { selected }\end{array}$ & None & Placebo & $\begin{array}{l}\mathrm{Ne}=44 \\
\mathrm{Nc}=24\end{array}$ & $\mathrm{~T}: 6.0 \%$ & SWB: PA & - \\
\hline $\begin{array}{l}\text { Spence } 2007 \\
\text { [81] }\end{array}$ & $\begin{array}{l}\text { Life coaching and } \\
\text { attainment of goals }\end{array}$ & $10,10 \mathrm{w}$ & 38.6 & Individual & Community & $\begin{array}{l}\text { Self- } \\
\text { selected }\end{array}$ & None & Waiting list & $\begin{array}{l}\mathrm{Ne}=20 \\
\mathrm{Nc}=17\end{array}$ & $\begin{array}{l}4.8 \% \\
15.0 \% \\
\text { T: } 9.8 \%\end{array}$ & $\begin{array}{l}\text { SWB: } \\
\text { SWLS, PA } \\
\text { PWB: } \\
\text { RPWB }\end{array}$ & - \\
\hline $\begin{array}{l}\text { Wing } 2006 \\
\text { [71] }\end{array}$ & Positive writing & $3,3 d$ & $\begin{array}{l}40.3 \\
(18-79)\end{array}$ & Self-help & Community & $\begin{array}{l}\text { Self- } \\
\text { selected }\end{array}$ & None & Placebo & $\begin{array}{l}\mathrm{Ne}=58 \\
\mathrm{Nc}=55\end{array}$ & $6.3 \%$ & SWB: SWLS & - \\
\hline
\end{tabular}

Abbreviations. U = Unknown; $\mathrm{Ne}=$ Number of subjects in experimental group; $\mathrm{Nc}=$ Number of subjects in control group; $\mathrm{T}=$ Total; $\mathrm{nr}=$ Not reported; $\mathrm{MDD}=\mathrm{Major}$ Depressive Disorder; $\mathrm{AD}=\mathrm{Anxiety}$ Disorder; $\mathrm{GAD}=$ Generalized Anxiety Disorder; AHI = Authentic Happiness Inventory; EASQ = Expanded Attributional Style Questionnaire; GFI = Groningen Frailty Indicator; SWLS = Satisfaction with Life Scales; PA = Positive Affect; SHS $=$ State Hope Scale; PIL = Purpose in Life Test; CES-D = Center for Epidemiological Studies Depression Scale; SQ = Kellner's Symptom Questionnaire; CID = Clinical Interview for Depression; RPWB = Ryff's Scales of Psychological Well-being; MHC-SF = Mental Health Continuum-Short Form; HS = Hope Scale; BDI = Beck Depression Inventory; SPF-IL = Social Production Function-Index Level Scale; MS = Mastery Scale; AF-1 =

Affectometer 1; PCQ $=$ PsyCap Questionnaire; $P L A=$ Pleasant Affect; SHS(2) = Subjective Happiness Scale; GA = Global Appraisals of subjective well-being; PWI-A = Personal Well-being Index for Adults; OTH =
Orientations To Happiness; DASS-21 = Depression, Anxiety and Stress Scales; WB6 $=6$ well-being questions; SHI = Steen Happiness Index; PPTI = Positive Psychotherapy Inventory; HRSD = Hamilton Rating Scale for Depression; ZSRS = Zung Self-Rating Scale; LSI-A = Life Satisfaction Index-A; GDS = Geriatric Depression Scale; GSHS = Goal-Specific Hope Scale; NS = Needs Satisfaction 
$[29,51,82,83]$, in three studies in an organization $[62,78,79]$ and six studies recruited through the internet [30,52$55,63,69,74,84]$. Twenty-eight studies measured subjective well-being, 20 studied psychological well-being and 14 studied depressive symptoms. Half of the studies (20) were aimed at adult populations [29,30,33,51-56,62,63,65, $69,71,73,74,76,78,79,81-84]$. A substantial number of studies (17) were aimed at college students [29,32,34,51,58-61,64, $66-68,70,72,75,80,85]$ and two studies were aimed at older subjects $[57,77]$. In most studies (26) the PPI was delivered in the form of self-help [29,30,34,52-56,58,59,61,63-71,73$75,77,78,80,84,85]$. Eight studies used group PPIs [32,33,51, $57,60,62,72,76]$ and five used individual PPIs [51,79,81-83]. Intensity varied considerably across studies, ranging from a short one-day exercise [70] and a two-week self-help intervention $[65]$ to intensive therapy $[51,82,83]$ and coaching $[33,81]$.

\section{Post-test effects}

The random effect model showed that the PPIs were effective for all three outcomes. Results are presented in Table 3. The effect sizes of the individual studies at posttest are plotted in Figures 2, 3 and 4.

A composite moderate and statistically significant effect size (Cohen's $d$ ) was observed for subjective wellbeing $\mathrm{d}=0.34$ (95\% CI [0.22, 0.45], p<.01). For psychological well-being, Cohen's $d$ was 0.20 (95\% CI [0.09, $0.30], \mathrm{p}<.01$ ) and for depression $\mathrm{d}=0.23$ (95\% CI [0.09, 0.38 ], $\mathrm{p}<.01)$, which can be considered as small.

Heterogeneity was moderate for subjective well-being $\left(\mathrm{I}^{2}=49.5 \%\right)$ and depression $\left(\mathrm{I}^{2}=47.0 \%\right)$, and low for psychological well-being $\left(\mathrm{I}^{2}=29.0 \%\right)$. Effect sizes ranged from -0.09 [66] to 1.30 [64] for subjective well-being, -0.06 [78] to 2.4 [83] for psychological well-being and -0.17 [69] to 1.75 [83] for depression.

Removing outliers reduced effect sizes for all three outcomes: 0.26 (95\% CI [0.18, 0.33], $\mathrm{Z}=6.43, \mathrm{p}<.01$ ) for subjective well-being (Burton \& King, 2004 and Peters et al., 2010 removed) [64,70], 0.17 (95\% CI [0.09, 0.25], $\mathrm{Z}=4.18$, $\mathrm{p}<.01$ ) for psychological well-being (Fava et al. (2005) removed) [83] and 0.18 (95\% CI [0.07, 0.28], $\mathrm{Z}=3.33$, $\mathrm{p}<.01$ ) for depression (Fava, 2005 and Seligman, 2006 study 2 , removed) $[51,83]$. Removing the outliers reduced heterogeneity substantially (to a non-significant level).

\section{Follow-up effects}

Ten studies examined follow-up effects after at least three months and up to 12 months (Table 3). For the purposes of interpretation, we used only those studies examining effects from three to six months (short-term follow-up), thus excluding Fava et al. (2005) [83] which had a followup at one year. The random-effects model demonstrated small but significant effects in comparison with the control groups for subjective well-being (Cohen's $d$ 0.22, 95\% CI $[0.05,0.38], \mathrm{p}<.01)$ and for psychological well-being
(0.16, 95\% CI $[0.02,0.30], \mathrm{p}=.03)$. The effect was not significant for depression $(0.17,95 \%$ CI [-0.06, 0.39], $\mathrm{p}=.15)$. Heterogeneity was low for subjective well-being $\left(\mathrm{I}^{2}=1.1 \%\right)$ and psychological well-being $\left(\mathrm{I}^{2}=26.0 \%\right)$, and high for depression $\left(\mathrm{I}^{2}=63.9 \%\right)$.

\section{Subgroup analyses}

Subgroup analyses are presented in Table 4. We looked at self-selection, duration of the intervention, type of intervention, recruitment method, application of inclusion criteria related to certain psychosocial problems, and quality rating.

For depression, five out of six subgroups of studies resulted in significantly higher effect sizes. Higher effect sizes were found for 1) interventions of a longer duration (only in the meta regression analysis), 2) individual interventions, 3) studies involving referral from a health care practitioner or hospital, 4) studies which applied inclusion criteria based on psychosocial problems and 5) lower quality studies. For subjective well-being and psychological well-being, there were no significant differences between subgroups, although for the latter there was a recognizable trend in the same direction and on the same moderators, except for quality rating.

Twenty-six out of 39 studies were self-help interventions for which we conducted a separate subgroup analysis. However, there was little diversity within the self-help subgroup: only six studies examined intensive self-help for longer than four weeks, self-help was offered to people with specific psychosocial problems in only one study and more than half of the self-help studies $(n=14)$ recruited their participants via university. Consequently, there were no significant differences between subgroups for self-help interventions.

\section{Publication bias}

Indications for publication bias were found for all outcome measures, but to a lesser extent for subjective well-being. Funnel plots were asymmetrically distributed in such a way that the smaller studies often showed the more positive results (in other words, there is a certain lack of small insignificant studies). Orwin's fail-safe numbers based on a criterium effect size of 0.10 for subjective well-being (59), psychological well-being (16) and depression (13) were lower than required (respectively 150, 110 and 80). Egger's regression intercept also suggests that publication bias exists for psychological well-being (intercept $=1.18, \mathrm{t}=2.26, \mathrm{df}=18, \mathrm{p}=.04$ ) and depression (intercept $=1.45, \mathrm{t}=2.26, \mathrm{df}=12, \mathrm{p}=.03$ ), but not for subjective well-being (intercept $=1.20, t=1.55, \mathrm{df}=26$, $\mathrm{p}=0.13)$. The mean effect sizes of psychological wellbeing and depression were therefore recalculated by imputing missing studies using the Trim and Fill method. For psychological well-being, three studies were imputed 
Table 3 Main effects

\begin{tabular}{|c|c|c|c|c|c|c|}
\hline Outcome measures & $\mathrm{n}$ & $\mathrm{N}$ & Studies & Cohen's $d(95 \% \mathrm{Cl})$ & Heterogeneity & Test for overall effect \\
\hline \multicolumn{7}{|l|}{ Post-test } \\
\hline Subjective well-being & 28 & $\mathrm{Ne}=1449 \mathrm{Nc}=1265$ & {$[29,32-34,51,56-59,61,62,64,66-71,73,75,77,79-81,84,85]$} & $0.34(0.22-0.45)$ & $Q=53.5, d f=27, T^{2}=0.04(p<.01) ; l^{2}=49.5 \%$ & $Z=5.82(p<.01)$ \\
\hline Psychological well-being & 20 & $\mathrm{Ne}=2511 \mathrm{Nc}=977$ & {$[29,30,32,33,51-55,60,62,65,69,74-77,81-84]$} & $0.20(0.09-0.30)$ & $Q=26.8, d f=19, T^{2}=0.01 \quad(p=0.11) ; I^{2}=29.0 \%$ & $Z=3.65(p<.01)$ \\
\hline Depression & 14 & $\mathrm{Ne}=2435 \mathrm{Nc}=760$ & {$[30,51-55,61,63,69,74,76,80,82,83]$} & $0.23(0.09-0.38)$ & $Q=24.5, d f=13, T^{2}=0.03(p=0.03) ; I^{2}=47.0 \%$ & $Z=3.21(p<.01)$ \\
\hline \multicolumn{7}{|l|}{ Follow-up } \\
\hline Subjective well-being & 6 & $\mathrm{Ne}=329 \mathrm{Nc}=298$ & {$[51,57,62,67,69,77]$} & $0.22(0.05-0.38)$ & $Q=5.05, d f=5, T^{2}=0.00(p=0.41) ; I^{2}=1.1 \%$ & $Z=2.61(p<.01)$ \\
\hline Psychological well-being & 6 & $\mathrm{Ne}=1830 \mathrm{Nc}=417$ & {$[30,52-55,62,69,74,77]$} & $0.16(0.02-0.30)$ & $Q=6.8, d f=5, T^{2}=0.01 \quad(p=0.24) ; l^{2}=26.0 \%$ & $Z=2.20(p=.03)$ \\
\hline Depression & 5 & $\mathrm{Ne}=1765 \mathrm{Nc}=343$ & {$[30,51-55,69,74]$} & $0.17(-0.06-0.39)$ & $Q=11.1, d f=4, T^{2}=0.04(p=0.03) ; l^{2}=63.9 \%$ & $Z=1.44(p=.15)$ \\
\hline
\end{tabular}

$\mathrm{n}=$ Number of studies, $\mathrm{N}=$ Number of subjects, $\mathrm{Ne}=$ Number of subjects in experimental group; $\mathrm{Nc}=$ Number of subjects in control group . 


\section{Subjective well-being}

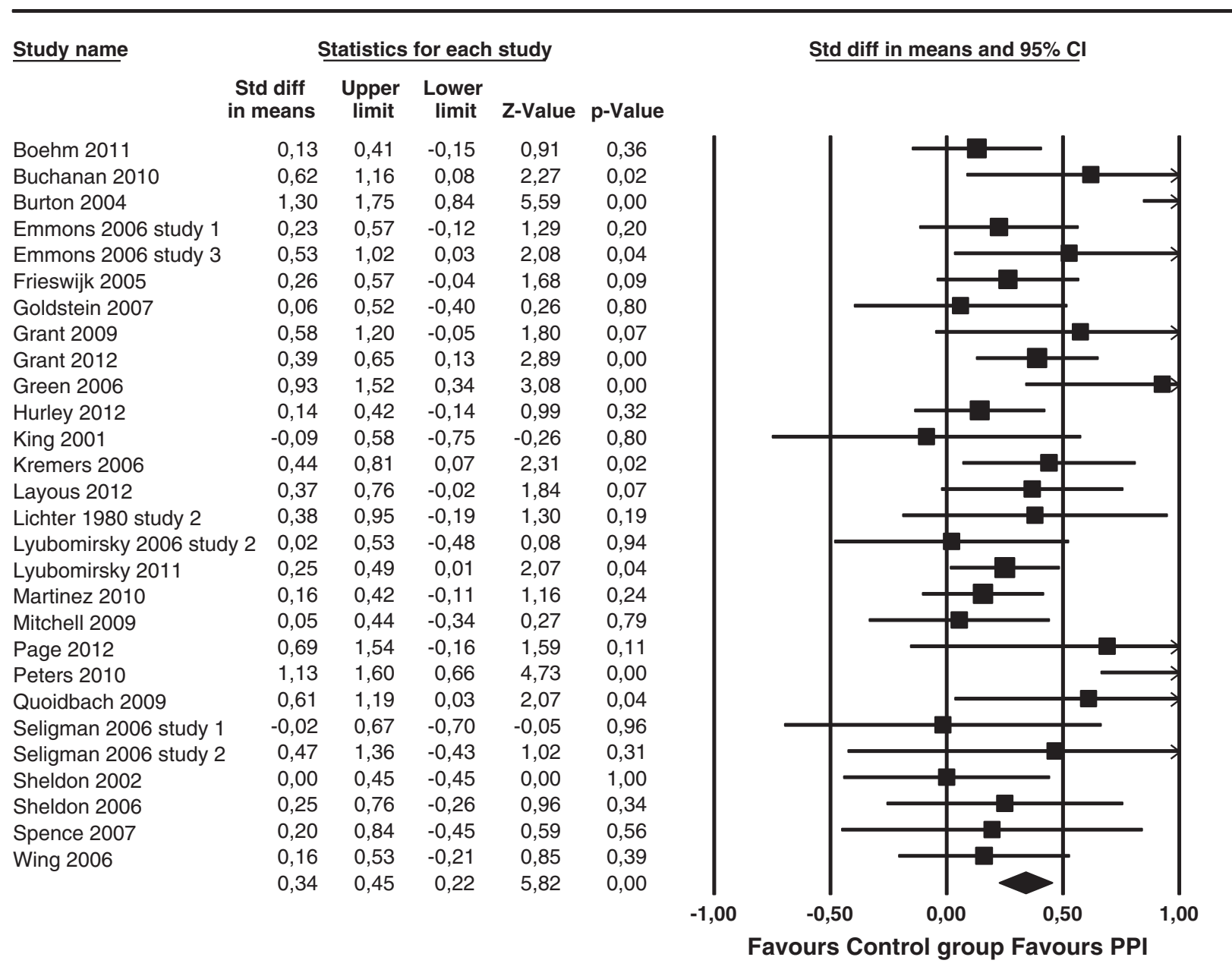

\section{Meta Analysis}

Figure 2 Post-test effects of positive psychology interventions on subjective well-being. The square boxes show effect size and sample size (the larger the box, the larger the sample size) in each study, and the line the 95\% confidence interval. The diamond reflects the pooled effect size and the width of the $95 \%$ confidence interval.

and the effect size was adjusted to 0.16 (95\% CI $0.03-$ 0.29). For depression, five studies were imputed and the adjusted effect size was 0.16 (95\% CI 0.00-0.32).

\section{Discussion}

\section{Main findings}

This meta-analysis synthesized effectiveness studies on positive psychology interventions. Following a systematic literature search, 40 articles describing 39 studies were included. Results showed that positive psychology interventions significantly enhance subjective and psychological well-being and reduce depressive symptoms. Effect sizes were in the small to moderate range. The mean effect size on subjective well-being was $0.34,0.20$ on psychological well-being, and 0.23 on depression. Effect sizes varied a great deal between studies, ranging from below 0 (indicating a negative effect) to 2.4 (indicating a very large effect). Moreover, at follow-up from three to six months, small but still significant effects were found for subjective well-being and psychological well-being, indicating that effects were partly sustained over time. These follow-up results should be treated with caution because of the small number of studies and the high attrition rates at follow-up.

Remarkably, effect sizes in the current meta-analysis are around 0.3 points lower than the effect sizes in the meta-analysis by Sin and Lyubomirsky (2009) [37]. We included a different set of studies in which the design 


\section{Psychological well-being}

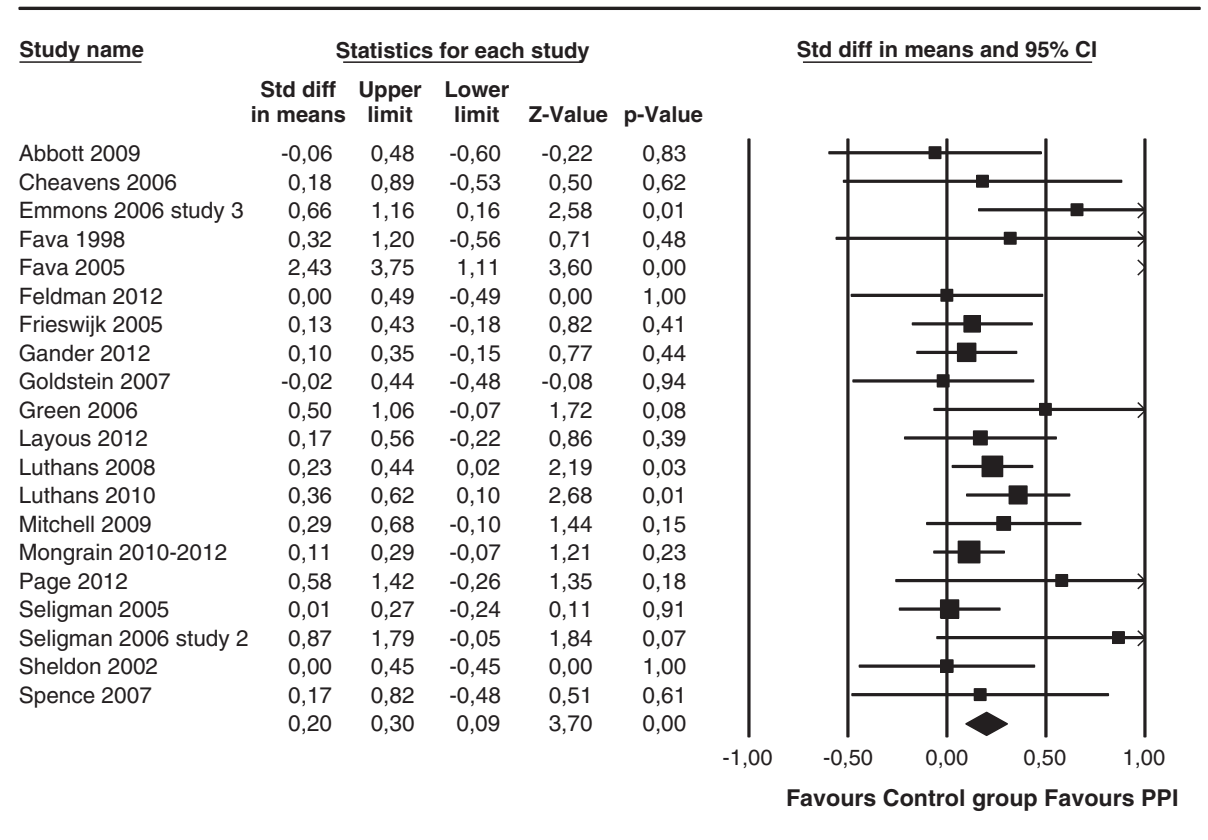

Meta Analysis

Figure 3 Post-test effects of positive psychology interventions on psychological well-being. The square boxes show effect size and sample size (the larger the box, the larger the sample size) in each study, and the line the 95\% confidence interval. The diamond reflects the pooled effect size and the width of the $95 \%$ confidence interval.

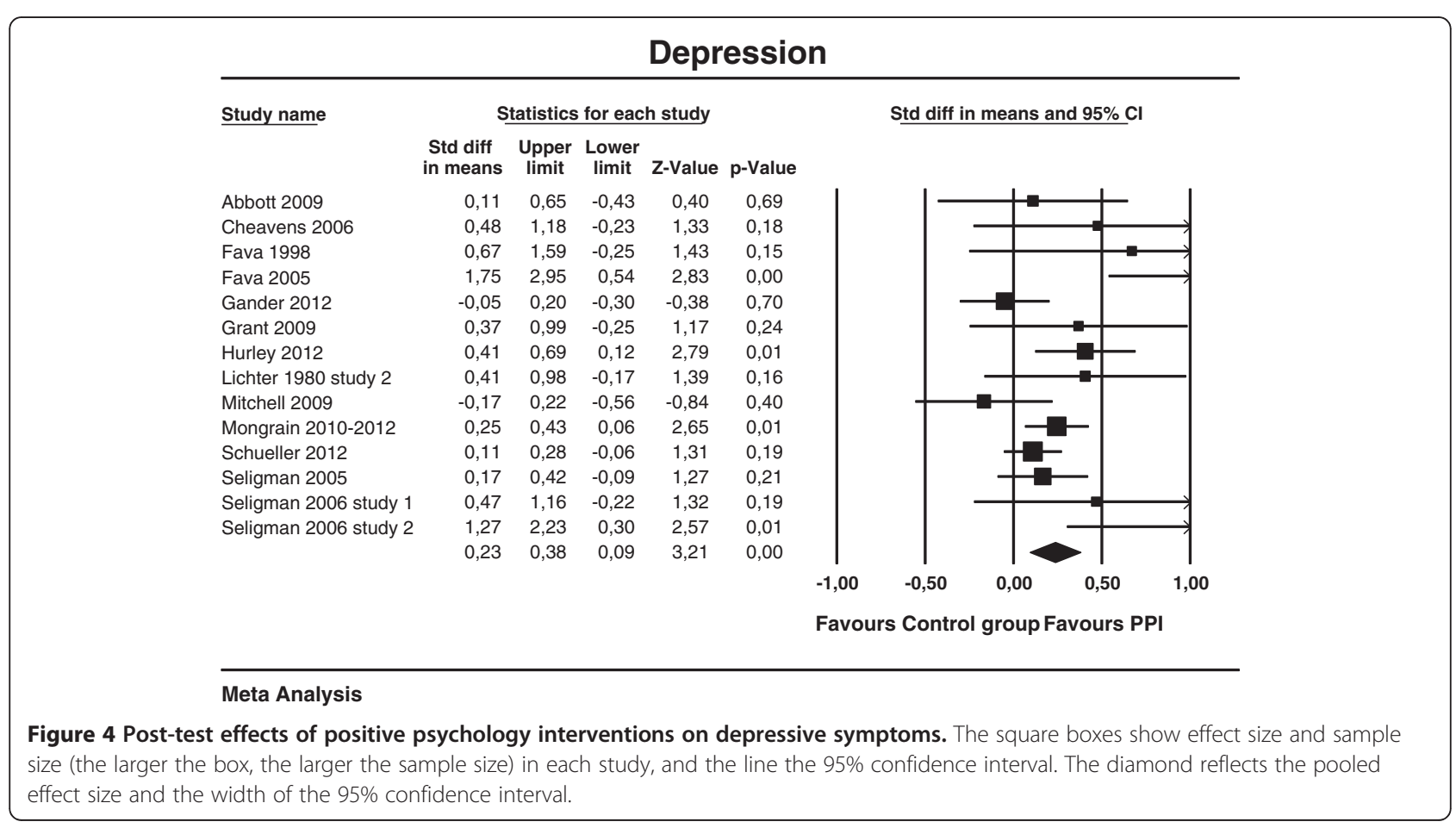


Table 4 Moderator effects: subgroup analysis (post-test)

\begin{tabular}{|c|c|c|c|c|c|}
\hline Outcome measure & Criteria & Subgroup (study) & $\mathrm{n}$ & Cohen's d $(95 \% \mathrm{Cl})$ & Test for subgroup differences \\
\hline \multirow[t]{18}{*}{ Subjective well-being } & \multirow[t]{2}{*}{ Self-selection } & Self-selected & 15 & $0.29(0.18-0.39)^{* * *}$ & \multirow[t]{2}{*}{$\mathrm{Q}=0.64, \mathrm{df}=1 \quad(\mathrm{p}=.43)$} \\
\hline & & Not self-selected & 13 & $0.38(0.17-0.60)^{* *}$ & \\
\hline & \multirow[t]{3}{*}{ Duration } & $<=4$ weeks & 17 & $0.35(0.18-0.52)^{* * *}$ & $\mathrm{Q}=1.84, \mathrm{df}=2(\mathrm{p}=.91)$ \\
\hline & & $<=8$ weeks & 6 & $0.24(0.10-0.39)^{* *}$ & \multirow[t]{2}{*}{ Slope $=0.01, Z=0.14(p=.89)$} \\
\hline & & $>8$ weeks & 5 & $0.43(0.17-0.68)^{* *}$ & \\
\hline & \multirow[t]{3}{*}{ Type } & Self help & 20 & $0.33(0.20-0.46)^{* * *}$ & \multirow[t]{3}{*}{$\mathrm{Q}=0.20, \mathrm{df}=2(\mathrm{p}=.91)$} \\
\hline & & Group & 5 & $0.38(0.03-0.73)^{*}$ & \\
\hline & & Individual & 3 & $0.41(0.01-0.81)^{*}$ & \\
\hline & \multirow[t]{5}{*}{ Recruitment } & Community & 6 & $0.29(0.11-0.48)^{* *}$ & \multirow[t]{5}{*}{$\mathrm{Q}=5.36, \mathrm{df}=4(\mathrm{p}=.25)$} \\
\hline & & Internet & 2 & $0.06(-0.24-0.35) \mathrm{ns}$ & \\
\hline & & Referral/hospital & 2 & $0.51(0.08-0.95)^{*}$ & \\
\hline & & University & 16 & $0.36(0.19-0.53)^{* * *}$ & \\
\hline & & Organization & 2 & $0.62(0.11-1.12)^{*}$ & \\
\hline & \multirow[t]{2}{*}{ Psychosocial problems } & Specific psychosocial problems & 4 & $0.31(0.09-0.52)^{* *}$ & \multirow[t]{2}{*}{$\mathrm{Q}=0.10, \mathrm{df}=1 \quad(\mathrm{p}=.76)$} \\
\hline & & None & 24 & $0.35(0.22-0.48)^{* * *}$ & \\
\hline & \multirow[t]{3}{*}{ Quality rating } & $\operatorname{Low}(1-2)$ & 16 & $0.29(0.17-0.40)^{* * *}$ & $\mathrm{Q}=2.41, \mathrm{df}=2(\mathrm{p}=.30)$ \\
\hline & & Medium (3-4) & 11 & $0.40(0.19-0.61)^{* * *}$ & \multirow[t]{2}{*}{ Slope $=-0.00, Z=0.08 \quad(p=.94)$} \\
\hline & & High (5-6) & 1 & $0.05(-0.34-0.44) \mathrm{ns}$ & \\
\hline \multirow[t]{18}{*}{ Psychological well-being } & \multirow[t]{2}{*}{ Self-selection } & Self-selected & 15 & $0.18(0.05-0.30)^{* *}$ & \multirow[t]{2}{*}{$\mathrm{Q}=0.32, \mathrm{df}=1 \quad(\mathrm{P}=.57)$} \\
\hline & & Not self-selected & 5 & $0.25(0.04-0.46) *$ & \\
\hline & \multirow[t]{3}{*}{ Duration } & $<=4$ weeks & 11 & $0.16(0.07-0.25)^{* * *}$ & $\mathrm{Q}=1.91, \mathrm{df}=2(\mathrm{p}=.39)$ \\
\hline & & $<=8$ weeks & 2 & $0.35(-0.20-0.89) \mathrm{ns}$ & \multirow{2}{*}{ Slope $=0.05, Z=0.95 \quad(p=.34)$} \\
\hline & & $>8$ weeks & 7 & $0.41(0.03-0.79)^{*}$ & \\
\hline & \multirow[t]{3}{*}{ Type } & Self help & 10 & $0.14(0.05-0.23)^{* *}$ & \multirow[t]{3}{*}{$\mathrm{Q}=3.76, \mathrm{df}=2(\mathrm{p}=.15)$} \\
\hline & & Group & 6 & $0.26(0.08-0.44)^{* *}$ & \\
\hline & & Individual & 4 & $0.81(-0.01-1.63) \mathrm{ns}$ & \\
\hline & \multirow[t]{5}{*}{ Recruitment } & Community & 4 & $0.20(-0.03-0.44) \mathrm{ns}$ & \multirow{5}{*}{$\mathrm{Q}=7.04, \mathrm{df}=4(\mathrm{p}=.13)$} \\
\hline & & Internet & 5 & $0.09(-0.03-0.21) \mathrm{ns}$ & \\
\hline & & Referral/hospital & 4 & $0.91(0.24-1.57)^{* *}$ & \\
\hline & & University & 5 & $0.22(0.08-0.35)^{* *}$ & \\
\hline & & Organization & 2 & $0.18(-0.43-0.78) \mathrm{ns}$ & \\
\hline & \multirow[t]{2}{*}{ Psychosocial problems } & Specific psychosocial problems & 5 & $0.59(0.00-1.18)^{*}$ & \multirow[t]{2}{*}{$Q=1.93, d f=1 \quad(p=.17)$} \\
\hline & & None & 15 & $0.17(0.08-0.25)^{* * *}$ & \\
\hline & Quality rating & $\operatorname{Low}(1-2)$ & 10 & $0.32(0.07-0.58)^{*}$ & $\mathrm{Q}=1.86, \mathrm{df}=2(\mathrm{p}=.40)$ \\
\hline & & Medium (3-4) & 9 & $0.15(0.06-0.24)^{* *}$ & Slope $=-0.01, Z=-0.45(p=.66)$ \\
\hline & & High (5-6) & 1 & $0.29(-0.11-0.68) \mathrm{ns}$ & \\
\hline Depression & Self-selection & Self-selected & 12 & $0.20(0.05-0.36)^{*}$ & $\mathrm{Q}=1.73, \mathrm{df}=1 \quad(\mathrm{p}=.19)$ \\
\hline & & Not self-selected & 2 & $0.41(0.15-0.66)^{* *}$ & \\
\hline & Duration & $<=4$ weeks & 7 & $0.15(0.02-0.28)^{*}$ & $\mathrm{Q}=4.86, \mathrm{df}=2(\mathrm{p}=.09)$ \\
\hline & & $<=8$ weeks & 2 & $0.47(-0.02-0.97) \mathrm{ns}$ & Slope $=0.20, Z=2.32(p=.02)$ \\
\hline & & $>8$ weeks & 5 & $0.68(0.15-1.21)^{*}$ & \\
\hline & Type & Self help & 8 & $0.15(0.03-0.27)^{*}$ & $\mathrm{Q}=6.99, \mathrm{df}=2(\mathrm{p}=.03)$ \\
\hline & & Group & 2 & $0.47(-0.02-0.97) \mathrm{ns}$ & \\
\hline
\end{tabular}


Table 4 Moderator effects: subgroup analysis (post-test) (Continued)

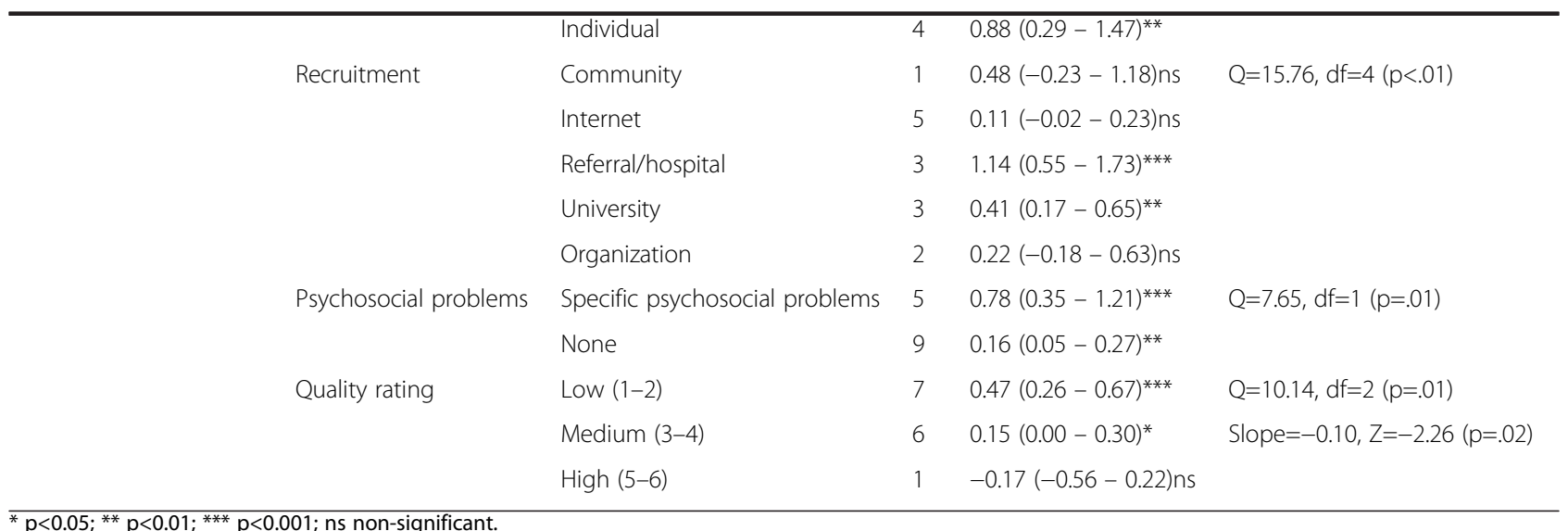

$\mathrm{n}=$ Number of studies.

quality was assured using randomized controlled trials only. Effectiveness research in psychotherapy shows that effect sizes are relatively small in high-quality studies compared with low-quality studies [35] and this might also be true for positive psychology interventions. In addition, we applied stricter inclusion criteria than those used by Sin and Lyubomirsky (2009) and therefore did not include studies on any related areas such as mindfulness and life review therapy. These types of interventions stem from long-standing independent research traditions for which effectiveness has already been established in several meta-analyses $[40,41]$. Also, the most recent studies were included. This might explain the overestimation of effect sizes in the meta-analysis by Sin and Lyubomirsky (2009).

Several characteristics of the study moderated the effect on depressive symptoms. Larger effects were found in interventions with a longer duration, in individual interventions (compared with self-help), when the interventions were offered to people with certain psychosocial problems and when recruitment was carried out via referral from a health care professional or hospital. Quality rating also moderated the effect on depression: the higher the quality, the smaller the effect. Interestingly, these characteristics did not significantly moderate subjective well-being and psychological well-being. However, there was a trend in the moderation of psychological well-being that was the same as that observed in the studies which included depression as an outcome. In general, effectiveness was increased when interventions were offered over a longer period, face-to-face on an individual basis in people experiencing psychosocial problems and when participants were recruited via the health care system.

Although it is clear that more intensive and face-toface interventions generate larger effects, the effects of short-term self-help interventions are small but significant. From a public health perspective, self-help interventions can serve as cost-effective mental health promotion tools to reach large target groups which may not otherwise be reached [86-88]. Even interventions presenting small effect sizes can in theory have a major impact on populations' well-being when many people are reached [89]. The majority of positive psychology interventions (in our study 26 out of 39 studies) are already delivered in a self-help format, sometimes in conjunction with face-to-face instruction and support. Apparently, self-help suits the goals of positive psychology very well and it would be very interesting to learn more about how to improve the effectiveness of PPI selfhelp interventions. However, a separate subgroup analysis on the self-help subgroup revealed no significant differences in the present meta-analysis. There was very little variation in the subgroups as regards population, duration of the intervention and recruitment method. As a result, this analysis does not give firm indications on how to improve the effectiveness of self-help interventions. It is possible that self-help could be enhanced by offering interventions to people with specific psychosocial problems, increasing the intensity of the intervention and embedding the interventions in the health care system. However, more studies in diverse populations, settings and with varying intensity are needed before we can begin to derive recommendations from this type of meta-analysis. Other research gives several additional indications on how to boost the efficacy of self-help interventions. Adherence tends to be quite low in self-help interventions [90,91] and therefore, enhancing adherence could be a major factor in improving effectiveness. Selfhelp often takes a 'one size fits all' approach, which may not be appropriate for a large group of people who will, as a consequence, not fully adhere to the intervention. 
Personalization and tailoring self-help interventions to individual needs [92] as well interactive support [93] might contribute to increased adherence and likewise improved effectiveness of (internet) self-help interventions.

\section{Study limitations}

This study has several limitations. First, the quality of the studies was not high, and no study met all of our quality criteria. For example, the randomization procedure was unclear in many studies. Also, most studies conducted completers-only analysis, as opposed to intention-to-treat analysis. This could have seriously biased the results [35]. However, the low quality of the studies could have been overstated as the criteria were scored conservatively: we gave a negative score when a criterion was not reported. Even so, more high-quality randomized-controlled trials are needed to enable more robust conclusions about the effects of PPIs. Second, different types of interventions are lumped together as positive psychology interventions, despite the strict inclusion criteria we applied. As expected, we found a rather high level of heterogeneity. In the future, it might be wise and meaningful to conduct metaanalyses that are restricted to specific types of interventions, for example gratitude interventions, strengths-based interventions and well-being therapy, just as has already been carried out with, for example, mindfulness and life review. In the present meta-analysis, studies on these specific interventions were too small and too diverse to allow for a subgroup-analysis. Third, the exclusion of non peerreviewed articles and grey literature could have led to bias, and possibly also to the publication bias we found in our study. Fourth, although we included a relatively large number of studies in the meta-analysis, the number of studies in some subgroups was still small. Again, more randomized-controlled trials are needed to draw firmer conclusions. Sixth, the study of positive education is an emerging field in positive psychology [94-98] but school-based interventions were excluded from our metaanalysis due to the strict application of the inclusion criteria (only studies with randomization at individual level were included).

\section{Conclusion}

This meta-analysis demonstrates that positive psychology interventions can be effective in the enhancement of subjective and psychological well-being and may help to reduce depressive symptom levels. Results indicate that the effects are partly sustained at short-term follow-up. Although effect sizes are smaller in our metaanalysis, these results can be seen as a confirmation of the earlier meta-analysis by Sin and Lyubomirsky (2009). Interpretation of our findings should take account of the limitations discussed above and the indications for publication bias.

\section{Implications for practice}

In mental health care PPIs can be used in conjunction with problem-based preventive interventions and treatment. This combination of interventions might be appropriate when clients are in remission; positive psychology interventions may then be used to strengthen psychological and social recourses, build up resilience and prepare for normal life again. On the basis of the moderator analysis, we would recommend the delivery of interventions over a longer period (at least four weeks and preferably eight weeks or longer) and on an individual basis. Practitioners can tailor their treatment strategy to the needs and preferences of a client and can use positive psychology exercises in combination with other evidence-based interventions that have a positive approach and aim to enhance well-being, such as mindfulness interventions [40], Acceptance and Commitment Therapy [7,99], forgiveness interventions [42], behavioral-activation [100] and reminiscence [41,101].

In the context of public health, positive psychology interventions can be used as preventive, easily accessible and non-stigmatizing tools. They can potentially be used in two ways: 1) in mental health promotion (e.g. leaflets distributed for free at community centers, (mental) health internet portals containing psycho-education), and 2) as a first step in a stepped care approach. In the stepped care model, clients start with a low-intensity intervention if possible, preferably a self-directed intervention. These interventions can be either guided by a professional or unguided, and are increasingly delivered over the internet. Clinical outcomes can be monitored and people can be provided with more intensive forms of treatment, or referred to specialized care, if the first-step intervention does not result in the desired outcome [102].

\section{Recommendations for research}

Regarding the research agenda, there is a need for more high-quality studies, and more studies in diverse (clinical) populations and diverse intervention formats to know what works for whom. Standards for reporting studies should also be given more attention, for example by reporting randomized controlled trials according to the CONSORT statement [103]. In addition, we encourage researchers to publish in peer-reviewed journals, even when the sample sizes are small or when there is a null finding of no effect, as this is likely to reduce the publication bias in positive psychology. Furthermore, most studies are conducted in North America. Therefore, replications are needed in other countries and cultures because some positive psychology concepts may require adaptation to other cultures and outlooks (e.g. see Martinez et al., 2010) [68]. Last but not least, we strongly recommend conducting cost-effectiveness studies aiming to establish the societal and public health impact of positive psychology interventions. This type of 
information is likely to help policy makers decide whether positive psychology interventions offer good value for money and should therefore be placed on the mental health agenda for the $21^{\text {st }}$ century.

\section{Competing interests}

The authors declare that they have no competing interests.

\section{Authors' contributions}

$L B$ conducted the meta-analysis, including the literature selection and dataanalysis, and wrote the manuscript. MH took care of selecting the articles and cross-checking the data. All authors contributed to the design of the study. EB, GW, HR and FS are advisors in the project. All authors provided comments and approved the final manuscript.

\section{Acknowledgements}

We are grateful to Toine Ketelaars and Angita Peterse for the literature search and Jan Walburg for his comments on the manuscript. We would also like to thank Deirdre Brophy for the English language edit.

\section{Author details}

'Department of Public Mental Health, Trimbos Institute, Netherlands Institute of Mental Health and Addiction, P.O. Box 7253500 AS, Utrecht, the Netherlands. ${ }^{2}$ Innovation Centre of Mental Health \& Technology, Trimbos Institute, Netherlands Institute of Mental Health and Addiction, Utrecht, The Netherlands. ${ }^{3}$ Department of Psychology, Health and Technology, University of Twente, Enschede, The Netherlands. ${ }^{4}$ The EMGO institute for Health and Care research, VU University, Amsterdam, The Netherlands. Innovation Incubator, Leuphana University, Lueneburg, Germany. ${ }^{6}$ Department of Epidemiology and Biostatistics, VU University Medical Centre, Amsterdam, The Netherlands.

Received: 22 June 2012 Accepted: 29 January 2013

Published: 8 February 2013

\section{References}

1. Rupke SJ, Blecke D, Renfrow M: Cognitive Therapy for Depression. Am Fam Physician 2006, 73:83-86.

2. Dobson KS: A meta-analysis of the efficacy of cognitive therapy for depression. J Consult Clin Psychol 1989, 57:414-419.

3. Cuijpers P, van Straten A, Warmerdam L: Problem solving therapies for depression: a meta-analysis. Eur Psychiatry 2007, 22:9-15.

4. Cuijpers P, Geraedts AS, van Oppen P, Andersson G, Markowitz JC, van Straten A: Interpersonal psychotherapy for depression: a meta-analysis. Am J Psychiatry 2011, 168:581-592.

5. Cuijpers P, Munoz RF, Clarke GN, Lewinsohn PM: Psychoeducational treatment and prevention of depression: the coping with depressioncourse thirty years later. Clin Psychol Rev 2009, 29:449-458.

6. Meulenbeek P, Willemse G, Smit F, van Balkom A, Spinhoven P, Cuijpers P: Early intervention in panic: pragmatic randomised controlled trial. $\mathrm{Br} J$ Psychiatry 2010, 196:326-331.

7. Fledderus M, Bohlmeijer E, Smit F, Westerhof GJ: Mental health promotion as a new goal in Public Mental Health Care: a randomized controlled trial of an intervention enhancing psychological flexibility. American Journal of Mental Health 2011, 100:2372-2378.

8. Bohlmeijer ET, Fledderus M, RokX TAJJ, Pieterse ME: Efficacy of an early intervention based on acceptance and commitment therapy for adults with depressive symptomatology: evaluation in a randomized controlled trial. Behav Res Ther 2011, 49:62-67.

9. WHO: Promoting mental health: Concepts, emerging evidence, practice (summary report). Geneva: World Health Organization; 2004.

10. Keyes CLM: Promoting and protecting mental health as flourishing: a complementary strategy for improving National Mental Health. Am Psychol 2007, 62:95-108.

11. Bergsma A, ten Have M, Veenhoven R, De Graaf R: Most people with mental disorders are happy. J Posit Psychol 2011, 6:253-259.

12. Diener E, Suh EM, Lucas RE, Smith HL: Subjective well-being: three decades of progress. Psychol Bull 1999, 125:276-302.

13. Ryff CD: Happiness is everything, or is it? Explorations on the meaning of psychological well-being. J Pers Soc Psychol 1989, 57:1069-1081.
14. Luthans F, Avolio BJ, Avey JB, Norman SM: Positive psychological capital: measurement and relationship with performance and satisfaction. Pers Psychol 2007, 60:541-572.

15. Keyes CLM, Grzywacz JG: Health as a complete state: the added value in work performance and healthcare costs. J Occup Environ Med 2005, 47:523-532.

16. Veenhoven R: Gezond geluk: Effecten van geluk op gezondheid en wat dat kan betekenen voor de preventieve gezondheidszorg (Healthy happiness: effect of happiness on health and what this could mean for preventive public health). Rotterdam: Erasmus Universiteit; 2006.

17. Howell RT, Kern ML, Lyubomirsky S: Health benefits: meta-analytically determining the impact of well-being on objective health outcomes. Health Psychological Review 2007, 1:83-136.

18. Diener $E$, Chan MY: Happy people live longer: subjective well-being contributes to health and longevity. Applied Psychology: Health and Well-being 2011, 3:1-43.

19. Lamers SMA, Bolier L, Westerhof GJ, Smit F, Bohlmeijer ET: The impact of emotional well-being on long-term recovery and survival in physical illness: a meta-analysis. J Behav Med 2012, 35:538-547.

20. Pressman SD, Cohen S: Does positive affect influence health? Psychol Bull 2005, 131:925-971.

21. Keyes CLM, Dhingra SS, Simoes EJ: Change in level of positive mental health as a predictor of future risk of mental health. Am J Public Health 2010, 100:2366-2371.

22. Wood AM, Joseph S: The absence of positive psychological (eudemonic) well-being as a risk factor for depression: a ten year cohort study. J Affect Disord 2010, 122:213-217.

23. Lamers S, Bolier L, Westerhof GJ, Smit F, Bohlmeijer E: The impact of emotional well-being on long-term recovery and survival in physical illness: a meta-analysis. J Behav Med 2012, 5:538-547.

24. Seligman MEP, Csikszentmihalyi M: Positive psychology: an introduction. Am Psychol 2000, 55:5-14.

25. Maslow AH: Toward a psychology of being. 2nd edition. Oxford England: D. Van Nostrand; 1968.

26. Veenhoven R: The utility of happiness. Soc Indic Res 1988, 20:333-354

27. Ryan RM, Deci EL: On happiness and human potentials: a review of research on hedonic and eudaimonic well-being. Annu Rev Psychol 2001, 52:141-166.

28. IPPA: www.ippanetwork.org. Mount Royal, New Jersey: International Positive Psychology Association; 2009.

29. Emmons RA, McCullough ME: Counting blessings versus burdens: an experimental investigation of gratitude and subjective well-being in daily life. J Pers Soc Psychol 2003, 84:377-389.

30. Seligman MEP, Steen TA, Park N, Peterson C: Positive psychology progress: empirical validation of interventions. Am Psychol 2005, 60:410-421.

31. Otake K, Shimai S, Tanaka-Matsumi J, Otsui K, Fredrickson BL: Happy people become happier through kindness: a counting kindnesses intervention. J Happiness Stud 2006, 7:361-375.

32. Sheldon KM, Kasser T, Smith K, Share T: Personal goals and psychological growth: testing an intervention to enhance goal attainment and personality integration. Journal of Personality S2-Character \& Personality; A Quarterly for Psychodiagnostic \& Allied Studies 2002, 70:5-31.

33. Green LS, Oades LG, Grant AM: Cognitive-behavioral, solution-focused life coaching: enhancing goal striving, well-being, and hope. J Posit Psychol 2006, 1:142-149.

34. Sheldon KM, Lyubomirsky S: How to increase and sustain positive emotion: the effects of expressing gratitude and visualizing best possible selves. J Posit Psychol 2006, 1:73-82.

35. Cuijpers $P$, van Straten A, Bohlmeijer E, Hollon SD, Andersson G: The effects of psychotherapy for adult depression are overestimated: a metaanalysis of study quality and effect size. Psychol Med: A Journal of Research in Psychiatry and the Allied Sciences 2010, 40:211-223.

36. Bohlmeijer E, Prenger R, Taal E, Cuijpers P: The effects of mindfulness-based stress reduction therapy on mental health of adults with a chronic medical disease: a meta-analysis. J Psychosom Res 2010, 68:539-544.

37. Sin NL, Lyubomirsky S: Enhancing well-being and alleviating depressive symptoms with positive psychology interventions: a practice-friendly meta-analysis. J Clin Psychol 2009, 65:467-487.

38. Mitchell J, Vella-Brodrick D, Klein B: Positive psychology and the internet: a mental health opportunity. Electronic J Appl Psychol 2010, 6:30-41.

39. Meyer MC, Van Woerkom M, Bakker AB: The added value of the positive: a literature review of positive psychology interventions in organizations. Eur J Work Organ Psychol 2012, In press. 
40. Grossman P, Niemann L, Schmidt S, Walach H: Mindfulness-based stress reduction and health benefits: a meta-analysis. J Psychosom Res 2004, 57:35-43.

41. Bohlmeijer $E$, Roemer M, Cuijpers P, Smit F: The effects of reminiscence on psychological well-being in older adults: a meta-analysis. Aging Ment Health 2007, 11:291-300

42. Lundahl BW, Taylor MJ, Stevenson R, Roberts KD: Process-based forgiveness interventions: a meta-analytic review. Res Soc Work Pract 2008, 18:465-478.

43. Higgins JPT, Green S: Cochrane Handbook for systematic reviews of interventions (4.2.5). Chichester: John Wiley; 2005.

44. Juni $P$, Witschi $A$, Bloch $R$, Egger M: The hazards of scoring the quality of clinical trials for meta-analysis. JAMA: The Journal Of The American Medical Association 1999, 282:1054-1060.

45. Lipsey MW, Wilson DB: The efficacy of psychological, educational, and behavioral treatment. Confirmation from meta-analysis. Am Psychol 1993, 48:1181-1209.

46. Higgins JPT, Thompson SG, Deeks JJ, Altman DG: Measuring inconsistency in meta-analyses. Br Med J 2003, 327:557-560.

47. Borenstein M, Hedges LV, Higgins JPT, Rothstein HR: Introduction to MetaAnalysis. 1st edition. West Sussex: John Wiley \& Sons; 2009.

48. Orwin RG: A fail-safe N for effect size in meta-analysis. J Educ Stat 1983, 8:157-159.

49. Duval S, Tweedie R: Trim and fill: a simple funnel-plot-based method of testing and adjusting for publication bias in meta-analysis. Biometrics 2000, 56:455-463.

50. Moher D, Liberati A, Tetzlaff J, Altman DG: Preferred reporting items for systematic reviews and meta-analyses: the PRISMA statement. PLoS Med 2009, 6:e1000097.

51. Seligman MEP, Rashid T, Parks AC: Positive psychotherapy. Am Psychol 2006, 61:774-788

52. Mongrain M, Anselmo-Matthews T: Do positive psychology exercises work? A replication of Seligman et al. (). J Clin Psychol 2012, 68:382-389.

53. Mongrain M, Chin J, Shapira L: Practicing compassion increases happiness and self-esteem. J Happiness Stud 2011, 12:963-981.

54. Sergeant S, Mongrain M: Are positive psychology exercises helpful for people with depressive personality styles? J Posit Psychol 2011, 6:260-272.

55. Shapira LB, Mongrain M: The benefits of self-compassion and optimism exercises for individuals vulnerable to depression. J Posit Psychol 2010, 5:377-389.

56. Buchanan KE, Bardi A: Acts of kindness and acts of novelty affect life satisfaction. J Soc Psychol 2010, 150:235-237.

57. Kremers IP, Steverink N, Albersnagel FA, Slaets JPJ: Improved selfmanagement ability and well-being in older women after a short group intervention. Aging Ment Health 2006, 10:476-484.

58. Lyubomirsky S, Sousa L, Dickerhoof R: The costs and benefits of writing talking, and thinking about life's triumphs and defeats. J Pers Soc Psychol 2006, 90:692-708.

59. Quoidbach J, Wood AM, Hansenne M: Back to the future: the effect of daily practice of mental time travel into the future on happiness and anxiety. J Posit Psychol 2009, 4:349-355.

60. Feldman D, Dreher D: can hope be changed in 90 minutes? Testing the efficacy of a single-session goal-pursuit intervention for college students. J Happiness Stud 2012, 13:745-759.

61. Hurley DB, Kwon P: Results of a study to increase savoring the moment: differential impact on positive and negative outcomes. $J$ Happiness Stud 2012, 13:579-588.

62. Page K, Vella-Brodrick D: The working for wellness program: RCT of an employee well-being intervention. J Happiness Stud 2012, :1-25. http://link. springer.com/article/10.1007\%2Fs10902-012-9366-y? LI=true.

63. Schueller MS, Parks CA: Disseminating self-help: positive psychology exercises in an online trial. J Med Internet Res 2012, 14:e63.

64. Burton $C M$, King LA: The health benefits of writing about intensely positive experiences. J Res Personal 2004, 38:150-163.

65. Luthans F, Avey JB, Patera JL: Experimental analysis of a web-based training intervention to develop positive psychological capital. Acad Manag Learn Educ 2008, 7:209-221.

66. King LA: The health benefits of writing about life goals. Pers Soc Psychol Bulletin 2001, 27:798-807.

67. Lyubomirsky S, Dickerhoof R, Boehm JK, Sheldon KM: Becoming happier takes both a will and a proper way: an experimental longitudinal intervention to boost well-being. Emotion 2011, 11:391-402.
68. Martinez MML, Avia MD, Hernandez-Lloreda MJ: The effects of counting blessings on subjective well-being: a gratitude intervention in a Spanish sample. Span J Psychol 2010, 13:886-896.

69. Mitchell J, Stanimirovic R, Klein B, Vella-Brodrick D: A randomised controlled trial of a self-guided internet intervention promoting wellbeing. Comput Hum Behav 2009, 25:749-760.

70. Peters ML, Flink IK, Boersma K, Linton SJ: Manipulating optimism: can imagining a best possible self be used to increase positive future expectancies? J Posit Psychol 2010, 5:204-211.

71. Wing JF, Schutte NS, Byrne B: The effect of positive writing on emotional intelligence and life satisfaction. J Clin Psychol 2006, 62:1291-1302.

72. Luthans F, Avey JB, Avolio BJ, Peterson SJ: The development and resulting performance impact of positive psychological capital. Hum Resour Dev Q 2010, 21:41-67.

73. Boehm JK, Lyubomirsky S, Sheldon KM: A longitudinal experimental study comparing the effectiveness of happiness-enhancing strategies in Anglo Americans and Asian Americans. Cognition \& Emotion 2011, 25:1263-1272.

74. Gander F, Proyer RT, Ruch W, Wyss T: Strength-based positive interventions: further evidence for their potential in enhancing wellbeing and alleviating depression. J Happiness Stud 2012, http://link springer.com/article/10.1007/s10902-012-9380-0.

75. Layous K, Nelson KS, Lyubomirsky S: What is the optimal way to deliver a positive activity intervention? The case of writing about one's best possible selves. J Happiness Stud 2012, Published Online 06 May 2012. http://link.springer.com/article/10.1007/s10902-012-9346-2.

76. Cheavens JS, Feldman DB, Gum A, Michael ST, Snyder CR: Hope therapy in a community sample: a pilot investigation. Soc Indic Res 2006, 77:61-78.

77. Frieswijk N, Steverink N, Buunk BP, Slaets JPJ: The effectiveness of a bibliotherapy in increasing the self-management ability of slightly to moderately frail older people. Patient Educ Couns 2006, 61:219-227.

78. Abbott JA, Klein B, Hamilton C, Rosenthal A: The impact of online resilience training for sales managers on wellbeing and performance. E-J Appl Psychol 2009, 5:89-95.

79. Grant AM, Curtayne L, Burton G: Executive coaching enhances goal attainment, resilience and workplace well-being: a randomised controlled study. J Posit Psychol 2009, 4:396-407.

80. Lichter S, Haye K, Kammann R: Increasing happiness through cognitive retraining. Psychologist: New Zealand; 1980:9.

81. Spence GB, Grant AM: Professional and peer life coaching and the enhancement of goal striving and well-being: an exploratory study. J Posit Psychol 2007, 2:185-194.

82. Fava GA, Rafanelli C, Cazzaro M, Conti S, Grandi S: Well-being therapy: a novel psychotherapeutic approach for residual symptoms of affective disorders. Psychol Med 1998, 28:475-480.

83. Fava GA, Ruini C, Rafanelli C, Finos L, Salmaso L, Mangelli L, et al: Wellbeing therapy of generalized anxiety disorder. Psychotherapy and Psychosomatics S2- Acta Psychotherapeutica 2005, 74:26-30.

84. Goldstein ED: Sacred moments: implications on well-being and stress. J Clin Psychol 2007, 63:1001-1019.

85. Grant AM: Making positive change: a randomized study comparing solution-focused vs. problem-focused coaching questions. J Systemic Therapies 2012, 31:21-35

86. Bergsma A: Do self-help books help? J Happiness Stud 2007, 9:341-360.

87. den Boer PCAM, Wiersma D, Van Den Bosch RJ: Why is self-help neglected in the treatment of emotional disorders? A meta-analysis. Psychol Med: A Journal of Research in Psychiatry and the Allied Sciences 2004, 34:959-971.

88. Parks AC, Schueller SM, Tasimi A: Increasing happiness in the general population: empirically supported self-help? In To appaer in: Oxford Handbook of Happiness. Edited by Boniwell I, David S. Oxford: Oxford University Press; 2012.

89. Huppert FA: A new approach to reducing disorder and improving wellbeing. Perspect Psychol Sci 2009, 4:108-111.

90. Christensen $H$, Griffiths KM, Farrer L: Adherence in internet interventions for anxiety and depression. J Med Internet Res 2009, 11:e13.

91. Schueller SM: Preferences for positive psychology exercises. J Posit Psychol 2010, 5:192-203.

92. Schueller SM: To each his own well-being boosting intervention: using preference to guide selection. J Posit Psychol 2011, 6:300-313. 
93. Cuijpers P, Donker T, van Straten A, Li J, Andersson G: Is guided self-help as effective as face-to-face psychotherapy for depression and anxiety disorders? A systematic review and meta-analysis of comparative outcome studies. Psychol Med: A Journal of Research in Psychiatry and the Allied Sciences 2010, 40:1943-1957.

94. Ruini C, Belaise C, Brombin C, Caffo E, Fava GA: Well-being therapy in school settings: a pilot study. Psychotherapy and Psychosomatics S2- Acta Psychotherapeutica 2006, 75:331-336.

95. Ruini C, Ottolini F, Tomba E, Belaise C, Albieri E, Visani D, et al: School intervention for promoting psychological well-being in adolescence. J Behav Ther Exp Psychiatry 2009, 40:522-532.

96. Wong MCS, Lee A, Sun J, Stewart D, Cheng FFK, Kan W, et al: A comparative study on resilience level between WHO health promoting schools and other schools among a Chinese population. Health Promot Int 2009, 24:149-155.

97. Gillham J, Reivich K: Cultivating optimism in childhood and adolescence. Ann Am Acad Pol Soc Sci 2004, 591:146-163.

98. Knoop HH: Education in 2025: How positive psychology can revitalize education. In Applied positive psychology: Improving everyday life, health, schools, work and society. Edited by Donaldson SI, Csikszentmihalyi M, Nakamura J. New York: Routledge; 2011.

99. Veehof MM, Oskam MJ, Schreurs KMG, Bohlmeijer ET: The effects of acceptance-based therapies on mental and physical health in chronic pain patients: results of a meta-analysis. Pain 2011, 152:533-542.

100. Mazzucchelli TG, Kane RT, Rees CS: Behavioral activation interventions for well-being: a meta-analysis. J Posit Psychol 2010, 5:105-121.

101. Korte J, Bohlmeijer ET, Cappeliez P, Smit F, Westerhof GJ: Life review therapy for older adults with moderate depressive symptomatology: a pragmatic randomized controlled trial. Psychol Med 2012, 42:1 163-1173.

102. van Straten A, Seekles W, van 't Veer-Tazelaar N, Beekman ATF, Cuijpers P: Stepped care for depression in primary care: what should be offered and how? Med J Australia 2010, 192:S36-S39.

103. Altman DG: Better reporting of randomized controlled trials: the CONSORT statement. Br Med J 1996, 313:570-571.

doi:10.1186/1471-2458-13-119

Cite this article as: Bolier et al:: Positive psychology interventions: a meta-analysis of randomized controlled studies. BMC Public Health 2013 13:119.

\section{Submit your next manuscript to BioMed Central and take full advantage of:}

- Convenient online submission

- Thorough peer review

- No space constraints or color figure charges

- Immediate publication on acceptance

- Inclusion in PubMed, CAS, Scopus and Google Scholar

- Research which is freely available for redistribution 